\title{
Hydrodynamic interactions and Brownian forces in colloidal suspensions: Coarse-graining over time and length scales
}

\author{
J. T. Padding ${ }^{1,2,3}$ and A. A. Louis ${ }^{1}$ \\ ${ }^{1}$ Department of Chemistry, Cambridge University, Lensfield Road, Cambridge CB2 1EW, United Kingdom \\ ${ }^{2}$ Schlumberger Cambridge Research, High Cross, Madingley Road, Cambridge CB3 OEL, United Kingdom \\ ${ }^{3}$ Computational Biophysics, University of Twente, P.O. Box 217, 7500 AE, Enschede, The Netherlands
}

(Received 13 March 2006; published 8 September 2006)

\begin{abstract}
We describe in detail how to implement a coarse-grained hybrid molecular dynamics and stochastic rotation dynamics simulation technique that captures the combined effects of Brownian and hydrodynamic forces in colloidal suspensions. The importance of carefully tuning the simulation parameters to correctly resolve the multiple time and length scales of this problem is emphasized. We systematically analyze how our coarsegraining scheme resolves dimensionless hydrodynamic numbers such as the Reynolds number Re, which indicates the importance of inertial effects, the Schmidt number Sc, which indicates whether momentum transport is liquidlike or gaslike, the Mach number, which measures compressibility effects, the Knudsen number, which describes the importance of noncontinuum molecular effects, and the Peclet number, which describes the relative effects of convective and diffusive transport. With these dimensionless numbers in the correct regime the many Brownian and hydrodynamic time scales can be telescoped together to maximize computational efficiency while still correctly resolving the physically relevant processes. We also show how to control a number of numerical artifacts, such as finite-size effects and solvent-induced attractive depletion interactions. When all these considerations are properly taken into account, the measured colloidal velocity autocorrelation functions and related self-diffusion and friction coefficients compare quantitatively with theoretical calculations. By contrast, these calculations demonstrate that, notwithstanding its seductive simplicity, the basic Langevin equation does a remarkably poor job of capturing the decay rate of the velocity autocorrelation function in the colloidal regime, strongly underestimating it at short times and strongly overestimating it at long times. Finally, we discuss in detail how to map the parameters of our method onto physical systems and from this extract more general lessons-keeping in mind that there is no such thing as a free lunch-that may be relevant for other coarse-graining schemes such as lattice Boltzmann or dissipative particle dynamics.
\end{abstract}

DOI: 10.1103/PhysRevE.74.031402

PACS number(s): 82.70.Dd, 05.40.-a, 47.11.-j, 47.20.Bp

\section{INTRODUCTION}

Calculating the nonequilibrium properties of colloidal suspensions is a highly nontrivial exercise because these depend both on the short-time thermal Brownian motion and the long-time hydrodynamic behavior of the solvent $[1,2]$. Moreover, the hydrodynamic interactions are of a manybody character and cannot usually be decomposed into a pairwise sum of intercolloid forces.

The fundamental difficulty of fully including the detailed solvent dynamics in computer simulations becomes apparent when considering the enormous time- and length-scale differences between mesoscopic colloidal and microscopic solvent particles. For example, a typical colloid of diameter $1 \mu \mathrm{m}$ will displace on the order of $10^{10}$ water molecules. Furthermore, a molecular dynamics (MD) scheme for the solvent would need to resolve time scales on the order of $10^{-15} \mathrm{~s}$ to describe the intermolecular forces, while a colloid of diameter $1 \mu \mathrm{m}$ in water diffuses over its own diameter in about $1 \mathrm{~s}$.

Clearly, simulating even an extremely crude molecular model for the solvent on the time scales of interest is completely out of the question: some form of coarse-graining is necessary. The object of this paper is to describe in detail one such scheme. But before we do so, we first briefly discuss a subset of the wide variety of different simulation techniques that have been devised to describe the dynamics of colloidal suspensions.
At the simplest level, the effects of the solvent can be taken into account through Brownian dynamics (BD) [3], which assumes that collisions with the solvent molecules induce a random displacement of the colloidal particle positions, as well as a local friction proportional to their velocity. Although, due to its simplicity, Brownian dynamics is understandably very popular, it completely neglects momentum transport through the solvent-as described by the NavierStokes equations-which leads to long-range hydrodynamic interactions (HI's) between the suspended particles. These HI's may fall off as slowly as $1 / r$ and can qualitatively affect the dynamical behavior of the suspension [1,2].

Beginning with the pioneering work of Ermak and McCammon [4], who added a simple representation of the Oseen tensor [1] to their implementation of $\mathrm{BD}$, many authors have applied computational approaches that include the HI's by an approximate analytical form. The most successful of these methods is Stokesian dynamics [5], which can take into account higher-order terms in a multipole expansion of the HI interactions. Although some more sophisticated recent implementations of Stokesian dynamics have achieved an $\mathcal{O}(N \ln N)$ scaling with the number of colloids [6], this method is still relatively slow and becomes difficult to implement in complex boundary conditions.

Another way to solve for the HI's is by direct numerical simulation (DNS) methods, where the solid particles are described by explicit boundary conditions for the Navier- 
Stokes equations [7]. These methods are better adapted to non-Brownian particles, but can still be applied to understand the effects of HI's on colloidal dynamics. The fluid particle dynamics (FPD) method of Tanaka and Araki [8] and a related method by Yamamoto and collaborators [9] are two important recent approaches to solving the Navier Stokes equations that go a step beyond standard DNS and simplify the problem of boundary conditions by using a smoothed step function at the colloid surfaces. In principle Brownian motion can be added to these methods [10].

The difficulty of including complex boundary conditions in DNS approaches has stimulated the use of lattice-gasbased techniques to resolve the Navier-Stokes equations. These methods exploit the fact that only a few conditions, such as (local) energy and momentum conservation, need to be satisfied to allow the correct (thermo)hydrodynamics to emerge in the continuum limit. Greatly simplified particle collision rules, easily amenable to efficient computer simulation, are therefore possible, and complex boundary conditions, such as those that characterize moving colloids, are easier to treat than in DNS methods. The most popular of these techniques is the lattice Boltzmann (LB) technique where a linearized and preaveraged Boltzmann equation is discretized and solved on a lattice [11]. Ladd has pioneered the application of this method to solid-fluid suspensions $[12,13]$. This is illustrated schematically in Fig. 1. The solid particles are modeled as hollow spheres, propagated with Newtonian dynamics and coupled to the LB solvent through a bounce-back collision rule on the surface. The fluctuations that lead to Brownian motion were modeled by adding a stochastic term to the stress tensor. Recently this has been criticized, and an improved method to include Brownian noise that does not suffer from some lattice induced artifacts was suggested [14]. A number of other groups have recently derived alternative ways of coupling a colloidal particle to a LB fluid [15-17], in part to simulate the dynamics of charged systems.

The discrete nature of lattice-based methods can also bring disadvantages, particularly when treating fluids with more than one length scale. Dissipative particle dynamics (DPD) $[18,19]$ is a popular off-lattice alternative that includes hydrodynamics and Brownian fluctuations. It can be viewed as an extension of standard Newtonian MD techniques, but with two important innovations: (i) soft potentials that allow large time steps and rapid equilibration and (ii) a Galilean-invariant thermostat that locally conserves momentum and therefore generates the correct Navier-Stokes hydrodynamics in the continuum limit.

In fact, these two methodological advances can be separated. Soft potentials such as those postulated for innovation (i) may indeed arise from careful equilibrium coarse-graining of complex fluids [20-23]. However, their proper statistical mechanical interpretation, even for equilibrium properties, is quite subtle. For example, a potential that generates the correct pair structure will not normally reproduce the correct virial pressure due to so called representability problems [24]. When used in dynamical simulations, the correct application of effective potentials is even more difficult to properly derive. For polymer dynamics, for instance, uncrossability constraints must be reintroduced to prevent coarse-

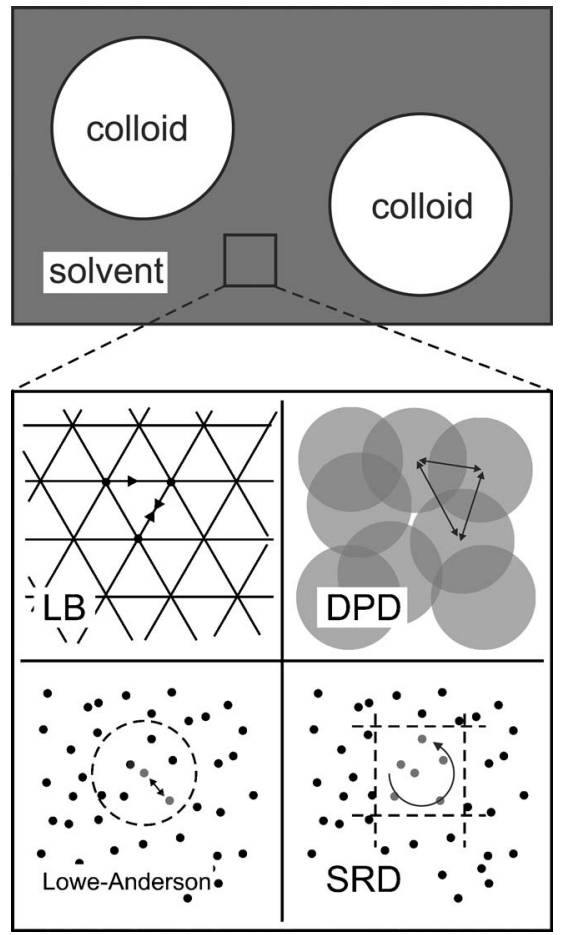

FIG. 1. Schematic picture depicting how a colloidal dispersion can be coarse-grained by replacing the solvent with a particle-based hydrodynamics solver such as lattice Boltzmann (LB), dissipative particle dynamics (DPD), the Lowe-Anderson thermostat, or stochastic rotation dynamics (SRD). Each method introduces an effective coarse-graining length scale that is chosen to be smaller than those of the mesoscopic colloids but much larger than the natural length scales of a microscopic solvent. By obeying local momentum conservation, these methods reproduce Navier-Stokes hydrodynamics on larger length scales. For LB, thermal fluctuations must be added in separately, but these emerge naturally for the other three methods. In this paper we focus on SRD, but many of the lessons learned should also apply to other particle based coarse-graining methods.

grained polymers from passing through one another [25]. Depletion interactions in a multicomponent solution also depend on the relative rates of depletant diffusion coefficients and particle flow velocities [26,27]. At present, therefore, the statistical mechanical origins of innovation (i) of DPD, the use of soft potentials out of equilibrium, are at best obscure. We are convinced that a correct microscopic derivation of the coarse-grained DPD representation of the dynamics, if this can indeed be done, will show that the interpretation of such soft potentials depends on dynamic as well as static (phase-space) averages. Viewing the DPD particles as static "clumps" of underlying fluid is almost certainly incorrect. It may, in fact, be more fruitful to abandon simple analogies to the potential energy of a Hamiltonian system and instead view the interactions as a kind of coarse-grained self-energy [28].

Innovation (ii), on the other hand, can be put on firmer statistical mechanical footing (see, e.g., [29]) and can be usefully employed to study the dynamics of complex systems with other types of interparticle interactions [30]. The main advantage of the DPD thermostat is that, by preserving mo- 
mentum conservation, the hydrodynamic interactions that intrinsically arise from microcanonical $\mathrm{MD}$ are preserved for calculations in the canonical ensemble. Other thermostats typically screen the hydrodynamic interactions beyond a certain length scale [31]. For weak damping this may not be a problem, but for strong damping it could be.

Simulating only the colloids with DPD ignores the dominant solvent hydrodynamics. While the solvent could be treated directly by DPD, as suggested in Fig. 1 (see also [32]), this method is quite computationally expensive because the "solvent" particles interact through pairwise potentials.

In an important paper [33], Lowe took these ideas further and combined the local momentum conservation of the DPD thermostat with the stochastic nature of the Anderson thermostat to derive a coarse-grained scheme, now called the Lowe-Anderson thermostat, which is much more efficient than treating the solvent with full DPD. It has recently been applied, for example, to polymer dynamics [34].

Independently, Malevanets and Kapral [35] derived a method now called stochastic rotation dynamics (SRD) or also multiple-particle collision dynamics (we choose the former nomenclature here). In many ways it resembles the Lowe-Anderson thermostat [33], or the much older direct simulation Monte Carlo (DSMC) method of Bird [36,37]. For all three of these methods, the particles are ideal and move in a continuous space, subject to Newton's laws of motion. At discrete time steps, a coarse-grained collision step allows particles to exchange momentum. In SRD, space is partitioned into a rectangular grid, and at discrete time steps the particles inside each cell exchange momentum by rotating their velocity vectors relative to the center of mass velocity of the cell (see Fig. 1). Similarly, in Lowe's method, a particle can, with a certain probability, exchange its relative velocity with another that lies within a certain radius. One can imagine other collision rules as well. As long as they locally conserve momentum and energy, just as the latticegas methods do, they generate the correct Navier-Stokes hydrodynamics, although for SRD it is necessary to include a grid-shift procedure to enforce Galilean invariance, something first pointed out by Ihle and Kroll [38]. An important advantage of SRD is that its simplified dynamics has allowed the analytic calculation of several transport coefficients [39-41], greatly facilitating its use. In the rest of this paper we will concentrate on the SRD method, although many of our general conclusions and derivations should be easily extendible to the Lowe-Anderson thermostat and related methods summarized in Fig. 1.

SRD can be applied to directly simulate flow, as done, for example, in Refs. [42,43], but its stochastic nature means that a noise average must be performed to calculate flow lines, and this may make it less efficient than preaveraged methods like LB. Where SRD becomes more attractive is for the simulation of complex particles embedded in a solvent. This is because in addition to long-range HI's, it also naturally contains Brownian fluctuations, and both typically need to be resolved for a proper statistical mechanical treatment of the dynamics of mesoscopic suspended particles. For example, SRD has been used to study polymers under flow [44-46], the effect of hydrodynamics on protein folding and polymer collapse [47], and the conformations of vesicles under flow [48]. In each of the previously mentioned examples, the suspended particles are coupled to the solvent by participating in the collision step.

A less coarse-grained coupling can be achieved by allowing direct collisions, obeying Newton's laws of motion, between the SRD solvent and the suspended particles. Such an approach is important for systems, such as colloidal suspensions, where the solvent and colloid time and length scales need to be clearly separated. Malevanets and Kapral [49] derived such a hybrid algorithm that combined a full MD scheme of the solute-solute and solute-solvent interactions, while treating the solvent-solvent interactions via SRD. Early applications were to a two-dimensional many-particle system [50] and to the aggregation of colloidal particles [51].

We have recently extended this approach and applied it to the sedimentation of up to 800 hard-sphere- (HS-) like colloids as a function of volume fraction, for a number of different values of the Peclet number [52]. To achieve these simulations we adapted the method of Malevanets and Kapral [49] in a number of ways that were only briefly described in Ref. [52]. In the current paper we provide a more in-depth analysis of the simulation method we used and describe some potential pitfalls. In particular we focus on the different time and length scales that arise from our coarsegraining approach, as well as the role of dimensionless hydrodynamic numbers that express the relative importance of competing physical phenomena. Very recently, another similar study of colloidal sedimentation and aggregation has been carried out [53]. Some of our results and analysis are similar, but some are different, and we will mention these where appropriate.

After the introductory overview above, we begin in Sec. II by carefully describing the properties of a pure SRD fluid, focusing on simple derivations that highlight the dominant physics involved for each transport coefficient. Section III explains how to implement the coupling of a colloidal particle to an SRD solvent bath and shows how to avoid spurious depletion interactions and how to understand lubrication forces. In Sec. IV we analyze how optimizing the efficiency of particle-based coarse-graining schemes affects different dimensionless hydrodynamic numbers, such as the Schmidt number, the Mach number, the Reynolds number, the Knudsen number, and the Peclet number. Section VI describes the hierarchy of time scales that determine the physics of a colloidal suspension and compares these to the compressed hierarchy in our coarse-grained method. In Sec. VII we tackle the question of how to map from a coarse-grained simulation, optimized for computational efficiency, to a real colloidal system. Finally after a Conclusions section, we include a few appendixes that discuss among other things how to thermostat the SRD system (Appendix A), why the popular Langevin equation without memory effects does a remarkably poor job of capturing both the long- and short-time dynamics of the colloidal velocity autocorrelation function (Appendix B), and, finally, how various physical properties and dimensionless numbers scale for an SRD simulation and how these compare to a colloid of radius $10 \mathrm{~nm}$ or $1 \mu \mathrm{m}$ in $\mathrm{H}_{2} \mathrm{O}$ (Appendix C). 


\section{PROPERTIES OF A PURE SRD SOLVENT}

In SRD, the solvent is represented by a large number $N_{f}$ of particles of mass $m_{f}$. Here and in the following, we will call these "fluid" particles, with the caveat that, however tempting, they should not be viewed as some kind of composite particles or clusters made up of the underlying (molecular) fluid. Instead, SRD should be interpreted as a Navier-Stokes solver that includes thermal noise. The particles are merely a convenient computational device to facilitate the coarse-graining of the fluid properties.

\section{A. Simulation method for a pure solvent}

In the first (propagation) step of the algorithm, the positions and velocities of the fluid particles are propagated for a time $\Delta t_{c}$ (the time between collision steps) by accurately integrating Newton's equations of motion,

$$
\begin{gathered}
m_{f} \frac{d \mathbf{v}_{i}}{d t}=\mathbf{f}_{i}, \\
\frac{d \mathbf{r}_{i}}{d t}=\mathbf{v}_{i},
\end{gathered}
$$

where $\mathbf{r}_{\mathbf{i}}$ and $\mathbf{v}_{\mathbf{i}}$ are the position and velocity of fluid particle $i$, respectively, while $\mathbf{f}_{\mathbf{i}}$ is the total (external) force on particle $i$, which may come from an external field such as gravity, fixed boundary conditions such as hard walls, or moving boundary conditions such as suspended colloids. The direct forces between pairs of fluid particles are, however, neglected in the propagation step. Herein lies the main advantage - the origin of the efficiency — of SRD. Instead of directly treating the interactions between the fluid particles, a coarse-grained collision step is performed at each time step $\Delta t_{c}$ : First, space is partitioned into cubic cells of volume $a_{0}^{3}$. Next, for each cell, the particle velocities relative to the center-of-mass velocity $\mathbf{v}_{\text {c.m. }}$ of the cell are rotated:

$$
\mathbf{v}_{i} \mapsto \mathbf{v}_{\text {c.m. }}+\mathbf{R}\left(\mathbf{v}_{i}-\mathbf{v}_{\text {c.m. }}\right) .
$$

$\mathbf{R}$ is a rotation matrix which rotates velocities by a fixed angle $\alpha$ around a randomly oriented axis. The aim of the collision step is to transfer momentum between the fluid particles while conserving the total momentum and energy of each cell. Both the collision and the streaming step conserve phase-space volume, and it has been shown that the singleparticle velocity distribution evolves to a MaxwellBoltzmann distribution [35].

The rotation procedure can thus be viewed as a coarsegraining of particle collisions over space and time. Because mass, momentum, and energy are conserved locally, the correct hydrodynamic (Navier-Stokes) equations are captured in the continuum limit, including the effect of thermal noise [35].

At low temperatures or small collision times $\Delta t_{c}$, the transport coefficients of SRD, as originally formulated [35], show anomalies caused by the fact that fluid particles in a given cell can remain in that cell and participate in several collision steps [38]. Under these circumstances the assumption of molecular chaos and Galilean invariance are incor- rect. However, this anomaly can be cured by applying a random shift of the cell coordinates before the collision step $[38,39]$.

It should also be noted that the collision step in SRD does not locally conserve angular momentum. As a consequence, the stress tensor $\sigma$ is not, in general, a symmetric function of the derivatives of the flow field (although it is still rotationally symmetric) [41]. The asymmetric part can be interpreted as a viscous stress associated with the vorticity $\boldsymbol{\nabla} \times \mathbf{v}$ of the velocity field $\mathbf{v}(\mathbf{r})$. The stress tensor will therefore depend on the amount of vorticity in the flow field. However, the total force on a fluid element is determined not by the stress tensor itself, but by its divergence $\boldsymbol{\nabla} \cdot \boldsymbol{\sigma}$, which is what enters the Navier-Stokes equations. Taking the divergence causes the explicit vorticity dependence to drop out (the gradient of a curl is zero). In principle, the stress tensor could be made symmetric by applying random rotations as well as random translations to the cell. Or, alternatively, angular momentum can be explicitly conserved by dynamically adapting the collision rule, as done by Ryder [54], who found no significant differences in fluid properties (although there is, of course, less flexibility in choosing simulation parameters). This result has been confirmed by some recent theoretical calculations [55], which demonstrate that the asymmetry of the stress tensor has only a few consequences, such as a correction to the sound wave attenuation associated with viscous dissipation of longitudinal density waves. These are not important for the fluid properties we are trying to model, and so, on balance, we chose not to implement possible fixes to improve on angular momentum conservation.

\section{B. Transport coefficients and the dimensionless mean free path}

The simplicity of SRD collisions has facilitated the analytical calculation of many transport coefficients [39-41,55]. These analytical expressions are particularly useful because they enable us to efficiently tune the viscosity and other properties of the fluid, without the need for trial-and-error simulations. In this section we will summarize a number of these transport coefficients, where possible giving a simple derivation of the dominant physics.

\section{Units and the dimensionless mean free path}

In this paper we will use the following units: lengths will be in units of cell size $a_{0}$, energies in units of $k_{B} T$, and masses in units of $m_{f}$ (this corresponds to setting $a_{0}=1$, $k_{B} T=1$, and $\left.m_{f}=1\right)$. Time, for example, is expressed in units of $t_{0}=a_{0} \sqrt{m_{f} / k_{B} T}$ and the number density $n_{f}=\gamma / a_{0}^{3}$, and other derived units can be found in Table I. We find it instructive to express the transport coefficients and other parameters of the SRD fluid in terms of the dimensionless mean free path

$$
\lambda=\frac{\Delta t_{c}}{a_{0}} \sqrt{\frac{k_{B} T}{m_{f}}}=\frac{\Delta t_{c}}{t_{0}},
$$

which provides a measure of the average fraction of a cell size that a fluid particle travels between collisions.

This particular choice of units helps highlight the basic physics of the coarse-graining method. The (nontrivial) ques- 
TABLE I. Units and simulation parameters for an SRD fluid. The parameters listed in the table all need to be fixed independently to determine a simulation.

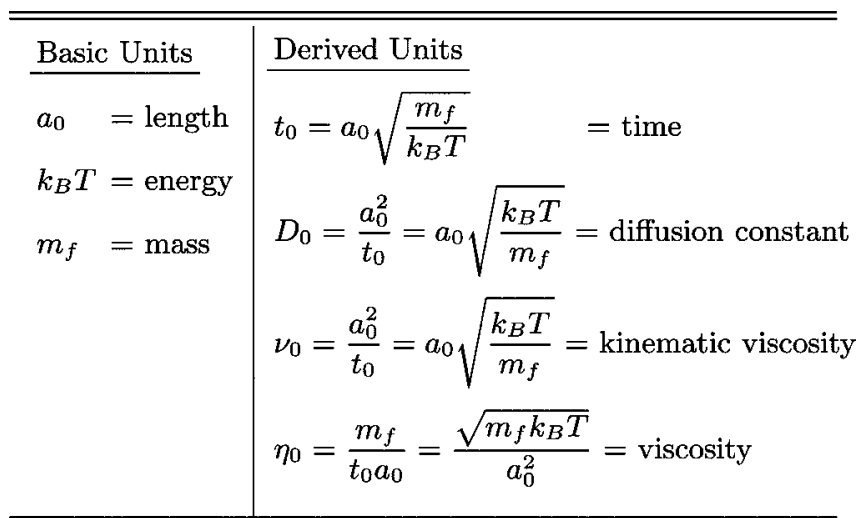

Independent fluid simulation parameters

$\gamma \quad=$ average number of particles per cell

$\Delta t_{c}=\mathrm{SRD}$ collision time step

$\alpha \quad=$ SRD rotation angle

$L=$ box length

Independent colloid simulation parameters

$$
\begin{aligned}
& \Delta t_{M D}=\text { MD time step } \\
& \sigma_{c c} \quad=\text { colloid-colloid collision diameter Eq. (16) } \\
& \epsilon_{c c} \quad=\text { colloid-colloid energy scale Eq. (16) } \\
& \sigma_{c f} \quad=\text { colloid-fluid collision diameter Eq. }(17) \\
& \epsilon_{c f} \quad=\text { colloid-fluid energy scale } \quad \text { Eq. }(17) \\
& N_{c} \quad=\text { number of colloids } \\
& M_{c} \quad=\text { colloid mass }
\end{aligned}
$$

tion of how to map them on to the units of real physical system will be discussed in Sec. VII.

\section{Fluid self-diffusion constant}

A simple back of the envelope estimate of the selfdiffusion constant $D_{f}$ of a fluid particle can be obtained from a random-walk picture. In a unit of time $t_{0}$, a particle will experience $1 / \lambda$ collisions, in between which it moves an average distance $\lambda a_{0}$. Similarly, a heavier (tagged) particle of mass $M_{t}$, which exchanges momentum with the fluid by participating in the coarse-grained collision step, will move an average distance $l_{M}=\Delta t_{c} \sqrt{k_{B} T / M_{t}}=\lambda a_{0} / \sqrt{M_{t}}$ between collisions. By viewing this motion as a random walk of step size $l_{M}$, the diffusion coefficient follows:

$$
\frac{D}{D_{0}} \sim \frac{m_{f}}{M_{t}} \lambda
$$

expressed in units of $D_{0}=a_{0}^{2} / t_{0}=a_{0} \sqrt{k_{B} T / m_{f}}$. The diffusion coefficient $D_{f}$ for a pure fluid particle of mass $m_{f}$ is therefore given by $D_{f} / D_{0} \approx \lambda$.

A more systematic derivation of the diffusion coefficient of a fluid particle, but still within a random-collision approximation, results in the following expression $[39,56]$ :

$$
\frac{D_{f}}{D_{0}}=\lambda\left[\frac{3}{2[1-\cos (\alpha)]}\left(\frac{\gamma}{\gamma-1}\right)-\frac{1}{2}\right] \text {. }
$$

The dependence on $\gamma$ is weak. If, for example, we take $\alpha$ $=\pi / 2$, the value used in this paper, then $\lim _{\gamma \rightarrow \infty} D_{f} / D_{0}=\lambda$, the same as Eq. (5).

A similar expression can be derived for the self-diffusion coefficient of a heavier tagged particle of mass $M_{t}[39,56]$ :

$$
\frac{D_{t}}{D_{0}}=\frac{\lambda m_{f}}{M_{t}}\left[\frac{3}{2[1-\cos (\alpha)]}\left(\frac{\gamma+M_{t} / m_{f}}{\gamma}\right)-\frac{1}{2}\right] \text {. }
$$

Note that this equation does not quite reduce to Eq. (6) for $M_{t} / m_{f}=1$ (because of slightly different approximations), but the relative discrepancy decreases with increasing $\gamma$.

While Eq. (6) is accurate for larger mean free paths, where the random-collision approximation is expected to be valid, it begins to show deviations from simulations for $\lambda$ $\lesssim 0.6$ [56], when longer-time kinetic correlations begin to develop. Ripoll et al. [56] argue that these correlations induce interactions of a hydrodynamic nature that enhance the diffusion coefficient for a fluid particle. For example, for $\lambda$ $=0.1$ and $\alpha=\frac{3}{4} \pi$, they measured a fluid self-diffusion constant $D_{f}$ that is about $25 \%$ larger than the value found from Eq. (6). The enhancement is even more pronounced for heavier particles: for the same simulation parameters they found that $D_{t}$ was enhanced by about $75 \%$ over the prediction of Eq. (7) when $M_{t} \gtrsim 10$.

We note that coupling a large particle to the solvent through participation in the coarse-grained collision step leads to a diffusion coefficient which scales with mass as $D_{t} / D_{0} \sim 1 / M_{t}$, whereas if one couples a colloid of radius $R_{c}$ to the solvent through direct MD collisions, one expects $D_{t} / D_{0} \sim a_{0} / R_{c} \sim\left(m_{f} / M_{t}\right)^{1 / 3}$. Moreover, it has been shown [56] that the effective hydrodynamic particle radius is approximately given by $a \approx 2 a_{0} /(\pi \gamma)$. For any reasonable average number of fluid particles per cell, the effective hydrodynamic radius $a$ of the heavier particle is therefore much less than the collision cell size $a_{0}$. On the other hand, the hydrodynamic field is accurately resolved down to a scale comparable to $a_{0}$ (vide infra). What this implies is that this coupling method will yield correct hydrodynamic interactions only at large distances, when the colloids are more than several hydrodynamic radii apart. All the above suggests that some care must be taken when interpreting the dynamics of heavier particles that couple through the coarse-grained collision step, especially when two or more heavy particles are in close proximity. 


\section{Kinematic viscosity}

The spread of a velocity fluctuation $\delta \mathbf{v}(\mathbf{r})$ in a fluid can be described by a diffusion equation [57]

$$
\frac{\partial \delta \mathbf{v}(\mathbf{r})}{\partial t}=\nu \nabla^{2} \delta \mathbf{v}(\mathbf{r})
$$

where $\nu$ is the kinematic viscosity, which determines the rate at which momentum or vorticity "diffuses away." The units of kinematic viscosity are $\nu_{0}=a_{0}^{2} / t_{0}=a_{0} \sqrt{k_{B} T / m_{f}}$ which are the same as those for particle self diffusion-i.e., $D_{0}=\nu_{0}$.

Momentum is transported through two mechanisms.

(i) By particles streaming between collision steps, leading to a "kinetic" contribution to the kinematic viscosity $\nu_{k i n}$. Since for this gaslike contribution the momentum is transported by particle motion, we expect $\nu_{k i n}$ to scale like the particle self-diffusion coefficient $D_{f}$-i.e., $\nu_{k i n} / \nu_{0} \sim \lambda$.

(ii) By momentum being redistributed among the particles of each cell during the collision step, resulting in a "collisional" contribution to the kinematic viscosity $\nu_{\text {col }}$. This mimics the way momentum is transferred due to interparticle collisions and would be the dominant contribution in a dense fluid such as water at standard temperature and pressure. Again a simple random-walk argument explains the expected scaling in SRD: Each collision step distributes momentum among particles in a cell, making a step size that scales like $a_{0}$. Since there are $1 / \lambda$ collision steps per unit time $t_{0}$, this suggests that the collisional contribution to the kinematic viscosity should scale as $\nu_{\text {col }} / \nu_{0} \sim 1 / \lambda$.

Accurate analytical expressions for the kinematic viscosity $\nu=\nu_{k i n}+\nu_{\text {col }}$ of SRD have been derived [40,55], and these can be rewritten in the dimensionless form

$$
\begin{gathered}
\frac{\nu_{\text {kin }}}{\nu_{0}}=\frac{\lambda}{3} f_{\text {kin }}^{\nu}(\gamma, \alpha), \\
\frac{\nu_{\mathrm{col}}}{\nu_{0}}=\frac{1}{18 \lambda} f_{\mathrm{col}}^{\nu}(\gamma, \alpha),
\end{gathered}
$$

where the dependence on the collisional angle $\alpha$ and fluid number density $\gamma$ is subsumed in the following two factors:

$$
f_{\text {kin }}^{\nu}(\gamma, \alpha)=\frac{15 \gamma}{\left(\gamma-1+e^{-\gamma}\right)[4-2 \cos (\alpha)-2 \cos (2 \alpha)]}-\frac{3}{2},
$$

$$
f_{\mathrm{col}}^{\nu}(\gamma, \alpha)=[1-\cos (\alpha)]\left(1-1 / \gamma+e^{-\gamma / \gamma}\right) .
$$

These factors only depend weakly on $\gamma$ for the typical parameters used in simulations. For example, at $\alpha=\pi / 2$ and $\gamma=5$, the angle and number densities we use in this paper, they take the values $f_{\text {kin }}^{\nu}(5, \pi / 2)=1.620$ and $f_{\text {col }}^{\nu}(5, \pi / 2)$ $=0.801$. For this choice of collision angle $\alpha$, they monotonically converge to $f_{\text {kin }}=f_{\text {col }}=1$ in the limit of large $\gamma$.

\section{Shear viscosity}

Suppose the fluid is sheared in the $x$ direction, with the gradient of the average flow field in the $y$ direction. Two neighboring fluid elements with different $y$ coordinates will then experience a friction force in the $x$ direction, as expressed by the $x y$ component of the stress tensor $\sigma$, which for a simple liquid is linearly proportional to the instantaneous flow field gradient,

$$
\sigma_{x y}=\eta \frac{\partial v_{x}(y)}{\partial y}
$$

The coefficient of proportionality $\eta$ is called the shear viscosity, and it is related to the kinematic viscosity by $\eta=\rho_{f} \nu$, where $\rho_{f}=m_{f} \gamma / a_{0}^{3}$ is the fluid mass density. From Eqs. (9)-(12) it follows that the two contributions to the shear viscosity can be written in dimensionless form as

$$
\begin{aligned}
& \frac{\eta_{\text {kin }}}{\eta_{0}}=\frac{\lambda \gamma}{3} f_{\text {kin }}^{\nu}(\gamma, \alpha), \\
& \frac{\eta_{\mathrm{col}}}{\eta_{0}}=\frac{\gamma}{18 \lambda} f_{\mathrm{col}}^{\nu}(\gamma, \alpha),
\end{aligned}
$$

where $\eta_{0}=m_{f} /\left(a_{0} t_{0}\right)=\sqrt{m_{f} k_{B} T} / a_{0}^{2}$ is the unit of shear viscosity.

In contrast to the expressions for the diffusion of a fluid particle or a tagged particle, Eqs. (9)-(15) compare quantitatively to simulations over a wide range of parameters $[39,40,56]$. For the parameters we used in our simulations in $[52,58]$-i.e., $\lambda=0.1, \alpha=\pi / 2$, and $\gamma=5$-the collisional contribution to the viscosity dominates: $\nu_{\text {kin }}=0.054 \nu_{0}$ and $\nu_{\text {col }}$ $=0.45 \nu_{0}$. This is typical for $\lambda \ll 1$, where $\nu$ and $\eta$ can be taken to a good first approximation by the collisional contribution only. In fact throughout this paper we will mainly focus on this small- $\lambda$ limit.

It is also instructive to compare the expressions derived in this section to what one would expect for simple gases, where, as famously first derived and demonstrated experimentally by Maxwell in the 1860s [59], the shear viscosity is independent of density. This result differs from the kinetic (gaslike) contribution to the viscosity in Eq. (14), because in a real dilute gas the mean free path scales as $\lambda \propto 1 / \gamma$, canceling the dominant density dependence in $\eta_{k i n} \propto \gamma \lambda$. The same argument explains why the self-diffusion and kinematic viscosity of the SRD fluid are, to first order, independent of $\gamma$, while in a gas they would scale as $1 / \gamma$. In SRD, the mean free path $\lambda$ and the density $\gamma$ can be varied independently. Moreover, the collisional contribution to the viscosity adds a new dimension, allowing a much wider range of physical fluids to be modeled than just simple gases.

\section{COLLOID SIMULATION METHOD}

Malevanets and Kapral [49] first showed how to implement a hybrid MD scheme that couples a set of colloids to a bath of SRD particles. In this section, we expand on their method, describing in detail the implementation we used in Ref. [52]. We restrict ourselves to HS-like colloids with steep interparticle repulsions, although attractions between colloids can easily be added on. The colloid-colloid and colloidfluid interactions $\varphi_{c c}(r)$ and $\varphi_{c f}(r)$, respectively, are integrated via a normal MD procedure, while the fluid-fluid interactions are coarse-grained with SRD. Because the num- 
ber of fluid particles vastly outnumbers the number of HS colloids, treating their interactions approximately via SRD greatly speeds up the simulation.

\section{A. Colloid-colloid and colloid-solvent interactions}

Although it is possible to implement an event-driven dynamics of HS colloids in an SRD solvent [60], here we approximate pure HS colloids by steep repulsive interactions of the Weeks-Chandler-Andersen (WCA) form [61]

$$
\varphi_{c c}(r)= \begin{cases}4 \epsilon_{c c}\left[\left(\frac{\sigma_{c c}}{r}\right)^{48}-\left(\frac{\sigma_{c c}}{r}\right)^{24}+\frac{1}{4}\right] & \left(r \leqslant 2^{1 / 24} \sigma_{c c}\right), \\ 0 & \left(r>2^{1 / 24} \sigma_{c c}\right) .\end{cases}
$$

Similarly, the colloid-fluid interaction takes the WCA form

$$
\varphi_{c f}(r)= \begin{cases}4 \epsilon_{c f}\left[\left(\frac{\sigma_{c f}}{r}\right)^{12}-\left(\frac{\sigma_{c f}}{r}\right)^{6}+\frac{1}{4}\right] & \left(r \leqslant 2^{1 / 6} \sigma_{c f}\right), \\ 0 & \left(r>2^{1 / 6} \sigma_{c f}\right) .\end{cases}
$$

The mass $m_{f}$ of a fluid particle is typically much smaller than the mass $M_{c}$ of a colloid, so that the average thermal velocity of the fluid particles is larger than that of the colloid particles by a factor of $\sqrt{M_{c} / m_{f}}$. For this reason the time step $\Delta t_{\mathrm{MD}}$ is usually restricted by the fluid-colloid interaction (17), allowing fairly large exponents $n$ for the colloid-colloid interaction $\varphi_{c c}(r)=4 \epsilon_{c c}\left[\left(\sigma_{c c} / r\right)^{2 n}-\left(\sigma_{c c} / r\right)^{n}+1 / 4\right]$. We choose $n=24$, which makes the colloid-colloid potential steep, more like hard spheres, while still soft enough to allow the time step to be set by the colloid-solvent interaction.

The positions and velocities of the colloidal spheres are propagated through the velocity Verlet algorithm [62] with a time step $\Delta t_{\mathrm{MD}}$ :

$$
\begin{gathered}
\mathbf{R}_{i}\left(t+\Delta t_{\mathrm{MD}}\right)=\mathbf{R}_{i}(t)+\mathbf{V}_{i}(t) \Delta t_{\mathrm{MD}}+\frac{\mathbf{F}_{i}(t)}{2 M_{c}} \Delta t_{\mathrm{MD}}^{2}, \\
\mathbf{V}_{i}\left(t+\Delta t_{\mathrm{MD}}\right)=\mathbf{V}_{i}(t)+\frac{\mathbf{F}_{i}(t)+\mathbf{F}_{i}\left(t+\Delta t_{\mathrm{MD}}\right)}{2 M_{c}} \Delta t_{\mathrm{MD}} .
\end{gathered}
$$

$\mathbf{R}_{i}$ and $\mathbf{V}_{i}$ are the position and velocity of colloid $i$, respectively. $\mathbf{F}_{i}$ is the total force on that colloid, exerted by the fluid particles, an external field, such as gravity, and external potentials such as repulsive walls, as well as other colloids within the range of the interaction potential (16).

The positions $\mathbf{r}_{i}$ and velocities $\mathbf{v}_{i}$ of SRD particles are updated by similarly solving Newton's Equations (1) and (2) every time step $\Delta t_{M D}$ and with the SRD procedure of Eq. (3) every time step $\Delta t_{c}$.

Choosing both $\Delta t_{M D}$ and $\Delta t_{c}$ as large as possible enhances the efficiency of a simulation. To first order, each time step is determined by different physics: $\Delta t_{M D}$ by the steepness of the potentials, and $\Delta t_{c}$ by the desired fluid properties, and so there is some freedom in choosing their relative values. We used $\Delta t_{c} / \Delta t_{\mathrm{MD}}=4$ for our simulations of sedimentation [52], but other authors have used ratios of 50 [49] or even an order

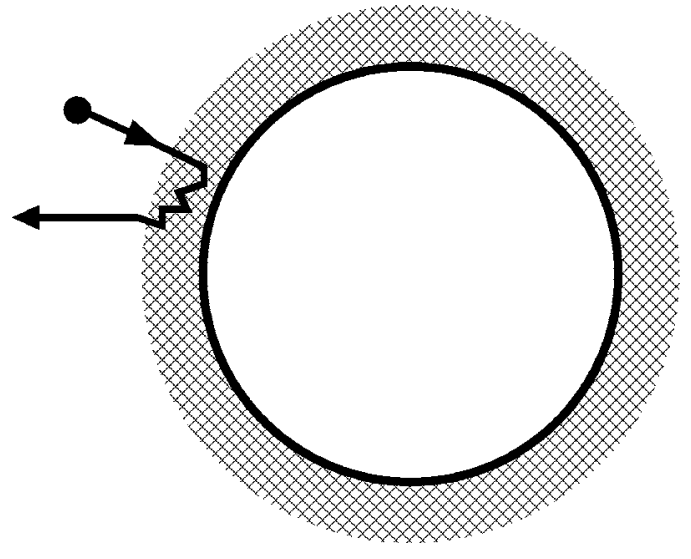

FIG. 2. Schematic picture depicting how a fluid molecule interacts with a colloid, imparting both linear and angular momentum. Near the colloidal surface, here represented by the shaded region, there may be a steric stabilization layer or a double-layer made up of co- and counter-ions. In SRD, the detailed manner in which a fluid particle interacts with this boundary layer is represented by a coarse-grained stick or slip boundary condition.

of magnitude larger than that [53]. Later in the paper we will reexamine this question, linking the time steps to various dimensionless hydrodynamic numbers and Brownian time scales.

\section{B. Stick and slip boundary conditions}

Because the surface of a colloid is never perfectly smooth, collisions with fluid particles transfer angular as well as linear momentum. As demonstrated in Fig. 2, the exact molecular details of the colloid-fluid interactions may be very complex and mediated via co- and counter-ions, grafted polymer brushes, etc. However, on the time and length scales over which our hybrid MD-SRD method coarse-grains the solvent, these interactions can be approximated by stick boundary conditions: the tangential velocity of the fluid, relative to the surface of the colloid, is zero at the surface of the colloid $[63,64]$. For most situations, this boundary condition should be sufficient, although in some cases, such as a nonwetting surface, large slip lengths may occur [65].

In computer simulations, stick boundary conditions may be implemented by bounce-back rules, where both parallel and perpendicular components of the relative velocity are reversed upon a collision with a surface. These have been applied by Lamura et al. [42], who needed to modify the bounce-back rules slightly to properly reproduce stick boundaries for a Poiseuille flow geometry.

Stick boundaries can also be modeled by a stochastic rule. After a collision, the relative tangential velocity $v_{t}$ and relative normal velocity $v_{n}$ are taken from the distributions

$$
\begin{gathered}
P\left(v_{n}\right) \propto v_{n} \exp \left(-\beta v_{n}^{2}\right), \\
P\left(v_{t}\right) \propto \exp \left(-\beta v_{t}^{2}\right),
\end{gathered}
$$

so that the colloid acts as an additional thermostat [66]. Such stochastic boundary conditions have been used for colloidal 
particles by Inoue et al. [50] and Hecht et al. [53]. We have systematically studied several implementations of stick boundary conditions for spherical colloids [58] and derived a version of the stochastic boundary conditions which reproduces linear and angular velocity correlation functions that agree with Enskog theory for short times and hydrodynamic mode-coupling theory for long times. We argue that the stochastic rule of Eq. (20) is more like a real physical colloidwhere fluid-surface interactions are mediated by steric stabilizing layers or local co- and counter-ion concentrationsthan bounce-back rules are [58].

Nevertheless, in this paper, many examples will be for radial interactions such as those described in Eq. (17). These do not transfer angular momentum to a spherical colloid and so induce effective slip boundary conditions. For many of the hydrodynamic effects we will discuss here the difference with stick boundary conditions is quantitative, not qualitative, and also well understood.

\section{Depletion and lubrication forces}

\section{Spurious depletion forces induced by the fluid}

We would like to issue a warning that the additional fluid degrees of freedom may inadvertently introduce depletion forces between the colloids. Because the number density of SRD particles is much higher than that of the colloids, even a small overlap between two colloids can lead to enormous attractions.

For low colloid densities the equilibrium depletion interaction between any two colloids caused by the presence of the ideal fluid particles is given by $[67,68]$

$$
\Phi_{\text {depl }}(d)=n_{f} k_{B} T\left[V_{\text {excl }}(d)-V_{\text {excl }}(\infty)\right],
$$

where $n_{f}=\gamma / a_{0}^{3}$ is the number density of fluid particles and $V_{\text {excl }}(d)$ is the (free) volume excluded from the fluid by the presence of two colloids separated by a distance $d$. The latter is given by

$$
V_{\mathrm{excl}}(d)=\int d^{3} \mathbf{r}\left\{1-\exp \left[-\beta \varphi_{c f}\left(\mathbf{r}-\mathbf{r}_{1}\right)-\beta \varphi_{c f}\left(\mathbf{r}-\mathbf{r}_{2}\right)\right]\right\},
$$

where $\left|\mathbf{r}_{1}-\mathbf{r}_{2}\right|=d$. An example is given in Fig. 3 where we have plotted the resulting depletion potential for the colloidsolvent interaction (17), with $\epsilon_{c c}=\epsilon_{c f}=2.5 k_{B} T$ as routinely used in our simulations, as well as the depletion interaction resulting from a truly HS colloid-solvent interaction. The latter can easily be calculated analytically, with the result

$$
\Phi_{\text {depl }}^{\mathrm{HS}}(d)=-n_{f} k_{B} T \frac{4}{3} \pi \sigma_{c f}^{3}\left(1-\frac{3 d}{4 \sigma_{c f}}+\frac{d^{3}}{16 \sigma_{c f}^{3}}\right) \quad \text { for } d<2 \sigma_{c f} .
$$

For the pure HS interactions, one could take $\sigma_{c c} \geqslant 2 \sigma_{c f}$ and the depletion forces would have no effect. But for interactions such as those used in Eqs. (16) and (17), the softer repulsions mean that intercolloid distances less than $\sigma_{c c}$ are regularly sampled. A more stringent criterion of $\sigma_{c c}$ must therefore be used to avoid spurious depletion effects. As an

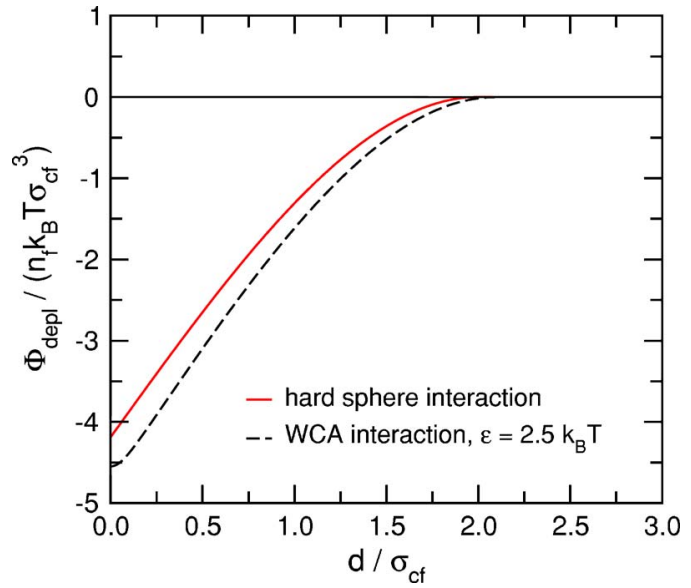

FIG. 3. (Color online) Effective depletion potentials induced between two colloids by the SRD fluid particles, for HS fluidcolloid (solid line) and WCA fluid-colloid (dashed line) interactions taken from Eq. (17). The interparticle distance is measured in units of $\sigma_{c f}$. Whether these attractive potentials have important effects or not depends on the choice of diameter $\sigma_{c c}$ in the bare colloidcolloid potential (16)

example, we reanalyze the simulations of Ref. [51], where a WCA form with $n=6$ was used for the fluid-colloid interactions and a normal Lennard Jones $n=6$ potential with $\sigma_{c f}$ $\geqslant \frac{1}{2} \sigma_{c c}$ was used for the colloid-colloid interactions. The authors found differences between "vacuum" calculations without SRD particles and a hybrid scheme coupling the colloids to an SRD solvent. These were correctly attributed to "solvent-induced pressure," which we quantify here as depletion interactions. For one set of their parameters, $\sigma_{c f}=\frac{1}{2} \sigma_{c c}$, the effect is mainly to soften the repulsion, but for their other parameter set, $\sigma_{c f}=0.65 \sigma_{c c}$, depletion attractions induce an effective attractive well depth of over $40 k_{B} T$.

Since the depletion potentials can be calculated analytically, one might try counteracting them by introducing a compensating repulsive potential of the form $\Phi_{\text {comp }}=-\Phi_{\text {depl }}$ between the colloids. However, there are three problems with this approach: First, at higher colloid packing fractions, third- and higher-order interactions may also need to be added, and these are very difficult to calculate. Second, the depletion interactions are not instantaneous and in fact only converge to their equilibrium average algebraically in time [26]. While this is not a problem for equilibrium properties, it will introduce errors for nonequilibrium properties. Finally, when external fields drive the colloid, small but persistent anisotropies in the solvent density around a colloid may occur [27]. Although these density variations are (and should be) small, the resulting variations in depletion interactions can be large.

To avoid these problems, we routinely choose the colloidfluid interaction range $\sigma_{c f}$ slightly below half the colloid diameter $\sigma_{c c} / 2$. More precisely, we ensure that the colloidcolloid interaction equals $2.5 k_{B} T$ at a distance $d$ where the depletion interactions have become zero-i.e., at a distance of twice the colloid-solvent interaction cutoff radius. Smaller distances will consequently be rare and adequately dealt with by the compensation potential. This solution may be a more 
realistic representation anyhow, since in practice for charge and even for sterically stabilized colloids, the effective colloid-colloid diameter $\sigma_{c c}$ is expected to be larger than twice the effective colloid-fluid diameter $\sigma_{c f}$. This is particularly so for charged colloids at large Debye screening lengths.

\section{Lubrication forces depend on surface details}

When two surfaces approach one another, they must displace the fluid between them, while if they move apart, fluid must flow into the space freed up between the surfaces. At very short intersurface distances, this results in so-called lubrication forces, which are repulsive for colloids approaching each other and attractive for colloids moving apart $[1,2,63]$. These forces are expected to be particularly important for driven dense colloidal suspensions; see, e.g., [69] for a recent review.

An additional advantage of our choice of diameters $\sigma_{c i}$ above is that more fluid particles will fit in the space between two colloids, and consequently the lubrication forces will be more accurately represented. It should be kept in mind that for colloids, the exact nature of the short-range lubrication forces will depend on physical details of the surface, such as its roughness or presence of a grafted polymeric stabilizing layer [70-72]. For perfectly smooth colloids, analytic limiting expressions for the lubrication forces can be derived [63], showing a divergence at short distances. We have confirmed that SRD resolves these lubrication forces down to surprisingly low interparticle distances. But at some point, this will break down, depending of course on the choice of simulation parameters (such as $\sigma_{c f} / a_{0}, \lambda$, and $\gamma$ ), as well as the details of the particular type of colloidal particles that one wishes to model [70-72]. An explicit analytic correction could be applied to properly resolve these forces for very small distances, as was recently implemented for lattice Boltzmann dynamics [73]. However, in this paper, we will assume that our choice of $\sigma_{c f}$ is small enough for SRD to sufficiently resolve lubrication forces. The lack of a complete divergence at very short distances may be a better model of what happens for real colloids anyway. For dense suspensions under strong shear, explicit lubrication force corrections as well as other short-ranged colloid-colloid interactions arising from surface details such as polymer coats will almost certainly need to be put in by hand; see, e.g., Ref. [72] for further discussion of this subtle problem.

\section{Test of static properties}

The equilibrium properties of a statistical mechanical system should be independent of the detailed dynamics by which it explores phase space. Therefore one requisite condition imposed on our coarse-grained dynamics is that it reproduces the correct static properties of an ensemble of colloids.

An obvious property to measure is the radial distribution function $g(r)$ [61]. In Fig. 4 we depict some $g(r)$ 's obtained from SRD simulations at a number of different densities and compare these to similar simulations with standard Brownian dynamics. The colloids interact via potentials of the form

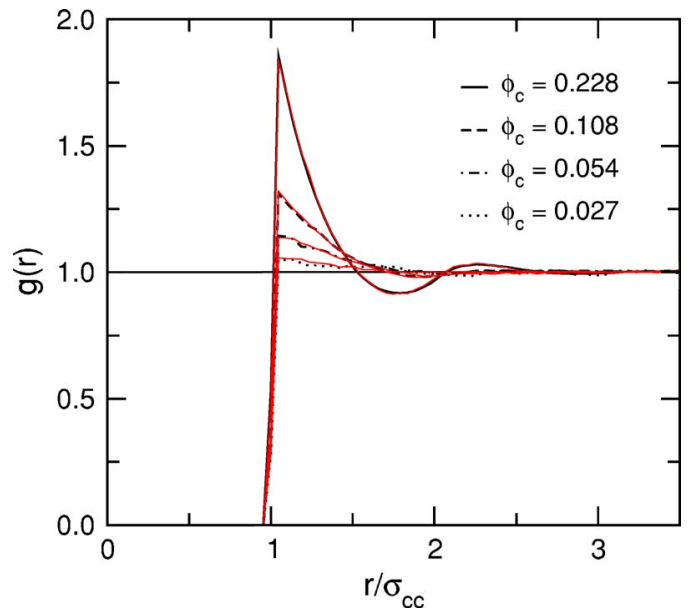

FIG. 4. (Color online) Colloid radial distribution functions $g(r)$ for various colloid volume fractions $\phi_{c}$. The simulations were performed with BD (black lines) and SRD (red lines) simulations, and are virtually indistinguishable within statistical errors.

(16) and (17) with $\sigma_{c f}=2 a_{0}, \sigma_{c c}=4.3 a_{0}$, and $\epsilon_{c c}=\epsilon_{c f}$ $=2.5 k_{B} T$. For the SRD we used $\gamma=5, \alpha=\frac{1}{2} \pi, \Delta t_{\mathrm{MD}}=0.025 t_{0}$, and $\Delta t_{c}=0.1 t_{0}$, implying $\lambda=0.1$. For the BD we used a background friction calculated from the effective hydrodynamic radius (vide infra) and a time step equal to the $\Delta t_{\mathrm{MD}}$ used above. The colloids were placed in a box of dimensions 32 $\times 32 \times 96 a_{0}^{3}$, and the number of particles was varied from 64 to 540 in order to achieve different packing fractions

$$
\phi_{c}=\frac{1}{6} \pi n_{c} \sigma_{c c}^{3},
$$

where the subscript in $\phi_{c}$ refers to volume fraction based on the colloid-colloid interaction, to distinguish it from the volume fraction $\phi$ based on the colloid hydrodynamic radius [74]. From Fig. 4 is clear that the two simulations are indistinguishable within statistical errors, as required. Even though $\sigma_{c f}<\frac{1}{2} \sigma_{c c}$, we still found it necessary to include an explicit compensating potential without which the $g(r)$ generated by SRD is increased noticeably at contact when compared to $\mathrm{BD}$ simulations.

Finally, we note that any simulation will show finite-size effects. These may be thermodynamic as well as dynamic. If there are thermodynamic finite-size effects (for example, due to a long correlation length caused by proximity to a critical point), then a correct simulation technique will show the same behavior regardless of the underlying dynamics.

\section{DIMENSIONLESS NUMBERS}

Different regimes of hydrodynamic behavior can be characterized by a series of dimensionless numbers that indicate the relative strengths of competing physical processes [57]. If two distinct physical systems can be described by the same set of hydrodynamic numbers, then their flow behavior will be governed by the same physics, even if their time and length scales differ by orders of magnitude. In other words, a macroscopic boulder sedimenting in viscous molten magma 
TABLE II. Dimensionless hydrodynamic numbers for colloidal suspensions.

\begin{tabular}{lccc}
\hline \hline Schmidt & collisional momentum transport & & Eq. (26) \\
\cline { 2 - 4 } Mach & $\frac{\mathrm{Sc}=\frac{\nu}{D_{f}}}{\text { sound velocity }}$ & $\mathrm{Ma}=\frac{v_{s}}{c_{s}}$ & Eq. (28) \\
Reynolds & $\frac{\text { inertial forces }}{\text { viscous forces }}$ & $\mathrm{Re}=\frac{v_{s} a}{\nu}$ & Eq. (29) \\
Knudsen & $\frac{\text { mean free path }}{\text { particle size }}$ & $\mathrm{Kn}=\frac{\lambda_{\text {free }}}{a}$ & Eq. (34) \\
Peclet & $\frac{\mathrm{convective} \mathrm{transport}}{\text { diffusive transport }}$ & $\mathrm{Pe}=\frac{v_{s} a}{D_{\text {col }}}$ & Eq. (35) \\
\hline \hline
\end{tabular}

and a mesoscopic colloid sedimenting in water may show similar behavior if they share key hydrodynamic dimensionless numbers. It is therefore very instructive to analyze where a system described by SRD sits in "hydrodynamic parameter space." In this section we review this parameter space, one "dimension" at a time, by exploring the hydrodynamic numbers listed in Table II.

\section{A. Schmidt number}

The Schmidt number

$$
\mathrm{Sc}=\frac{\nu}{D_{f}}
$$

is important for characterizing a pure fluid. It expresses the rate of diffusive momentum transfer, measured by the kinematic viscosity $\nu$, relative to the rate of diffusive mass transfer, measured by the fluid particle self-diffusion coefficient $D_{f}$. For a gas, momentum transport is dominated by mass diffusion, so that $\mathrm{Sc} \approx 1$, whereas for a liquid, momentum transport is dominated by interparticle collisions and $\mathrm{Sc} \gg 1$. For low Schmidt numbers, the dynamics of SRD is indeed "gaslike," while for larger Sc, the dynamics shows collective behavior reminiscent of the hydrodynamics of a liquid [56]. This distinction will be particularly important for simulating the dynamics of embedded particles that couple to the solvent through participation in the collision step. For particles that couple directly, either through a potential or through bounce-back or stochastic boundary conditions, this distinction is less important because the fluid-particle diffusion coefficient $D_{f}$ does not directly enter the Navier-Stokes equations. Of course other physical properties can be affected. For example, we expect that the self-diffusion coefficient of a colloid $D_{\text {col }}$ should be much smaller than the fluid diffusion coefficient-i.e., $D_{\text {col }} \ll D_{f}$ - to achieve the correct time-scale separation, as we will see in Sec. VI.

\section{Dependence of the Schmidt number on simulation parameters}

From Eqs. (6)-(12) it follows that the Schmidt number can be rewritten as

$$
\mathrm{Sc} \approx \frac{1}{3}+\frac{1}{18 \lambda^{2}}
$$

where we have ignored the dependence on $\gamma$ and $\alpha$ since the dominant scaling is with the dimensionless mean free path $\lambda$. For larger $\lambda$, where the kinetic contributions to the viscosity dominate, the Schmidt number is small and the dynamics is gaslike. Because both the fluid diffusion coefficient (6) and the kinetic contribution to the viscosity (9) scale linearly with $\lambda$, the only way to obtain a large Schmidt number is in the limit $\lambda \ll 1$ where the collisional contribution to $\nu$ dominates.

Low Schmidt numbers may be a more general characteristic of particle-based coarse-graining methods. For computational reasons, the number of particles is normally greatly reduced compared to the solvent one is modeling. Thus the average interparticle separation and mean free path are substantially increased, typically leading to a smaller kinematic viscosity and a larger fluid diffusion coefficient $D_{f}$. With $\mathrm{DPD}$, for example, one typically finds $\mathrm{Sc} \approx 1[29,33]$. It is therefore difficult to achieve large Schmidt numbers, as in a real liquid, without sacrificing computational efficiency. But this may not always be necessary. As long as momentum transport is clearly faster than mass transport, the solvent should behave in a liquidlike fashion. With this argument in mind, and also because the Schmidt number does not directly enter the Navier-Stokes equations we want to solve, we use $\lambda=0.1$ in this paper and in Ref. [52], which leads to a Sc $\approx 5$ for $\gamma=5$ and $\alpha=\pi / 2$. This should be sufficient for the problems we study. For other systems, such as those where the suspended particles are coupled to the solvent through the collision step, more care may be needed to ensure that the Schmidt number is indeed large enough [56].

\section{B. Mach number}

The Mach number measures the ratio

$$
\mathrm{Ma}=\frac{v_{s}}{c_{f}}
$$

between $v_{s}$, the speed of solvent or colloid flow, and $c_{f}$ $=\sqrt{(5 / 3)\left(k_{B} T / m_{f}\right)}$, the speed of sound. In contrast to the Schmidt number, which is an intrinsic property of the sol- 
vent, it depends directly on flow velocity. The Mach number measures compressibility effects [57] since sound speed is related to the compressibility of a liquid. Because $c_{f}$ in many liquids is of order $10^{3} \mathrm{~m} / \mathrm{s}$, the Mach numbers for physical colloidal systems are extremely small under normally achievable flow conditions. Just as for the Schmidt number, however, particle-based coarse-graining schemes drastically lower the Mach number. The particle mass $m_{f}$ is typically much greater than the mass of a molecule of the underlying fluid, resulting in lower velocities, and moreover, due to the lower density, collisions also occur less frequently. These effects mean that the speed of sound is much lower in a coarse-grained system than it is in the underlying physical fluid. Or, in other words, particle based coarse-graining systems are typically much more compressible than the solvents they model.

Mach number effects typically scale with $\mathrm{Ma}^{2}$ [57], and so the Mach number does not need to be nearly as small as for a realistic fluid to still be in the correct regime of hydrodynamic parameter space. This is convenient, because to lower the Mach number, one would need to integrate over longer fluid particle trajectories to allow, for example, a colloidal particle to flow over a given distance, making the simulation computationally more expensive. So there is a compromise between small Mach numbers and computational efficiency. We limit our Mach numbers to values such that $\mathrm{Ma} \leqslant 0.1$, but it might be possible, in some situations, to double or triple that limit without causing undue error. For example, incompressible hydrodynamics is used for aerodynamic flows up to such Mach numbers since the errors are expected to scale as $1 /\left(1-\mathrm{Ma}^{2}\right)$ [57]. When working in units of $m_{f}$ and $k_{B} T$, the only way to keep the Mach number below our upper limit is to restrict the maximum flow velocity to $v_{s} \lesssim 0.1 c_{f}$. The flow velocity itself is, of course, determined by the external fields, but also by other parameters of the system.

\section{Reynolds number}

The Reynolds number is one of the most important dimensionless numbers characterizing hydrodynamic flows. Mathematically, it measures the relative importance of the nonlinear terms in the Navier-Stokes equations [57]. Physically, it determines the relative importance of inertial over viscous forces and can be expressed as

$$
\operatorname{Re}=\frac{v_{s} a}{\nu},
$$

where $a$ is a length scale, in our case the hydrodynamic radius of a colloid-i.e., $a \approx \sigma_{c f}$-and $v_{s}$ is a flow velocity.

For a spherical particle in a flow, the following heuristic argument helps clarify the physics behind the Reynolds number: If the Stokes time

$$
t_{S}=\frac{\sigma_{c f}}{v_{s}},
$$

it takes a particle to advect over its own radius is about the same as the kinematic time

$$
\tau_{\nu}=\frac{\sigma_{c f}^{2}}{\nu}=\operatorname{Re}_{S}
$$

it takes momentum to diffuse over that distance-i.e., $\mathrm{Re}$ $=\tau_{\nu} / t_{S} \approx 1$-then the particle will feel vorticity effects from its own motion a distance $\sigma_{c f}$ away, leading to nonlinear inertial contributions to its motion. Since hydrodynamic interactions can decay as slowly as $1 / r$, their influence can be non-negligible. If, on the other hand, $\operatorname{Re} \ll 1$, then vorticity will have diffused away and the particle will only feel very weak hydrodynamic effects from its own motion.

Exactly when inertial finite-Reynolds-number effects become significant depends on the physical system under investigation. For example, in a pipe, where the length scale $a$ in Eq. (29) is its diameter, the transition from simpler laminar to more complex turbulent flow is at a pipe Reynolds number of $\operatorname{Re} \approx 2000[57,75]$. On the other hand, for a single spherical particle, a nonlinear dependence of the friction $\xi$ on the velocity $v_{s}$, induced by inertial effects, starts to become noticeable for a particle Reynolds number of $\operatorname{Re} \approx 1$ [57], while deviations in the symmetry of the streamlines around a rotating sphere have been observed in calculations for Re $\approx 0.1[76]$.

For typical colloidal suspensions, where the particle diameter is on the order of a few $\mu \mathrm{m}$ down to a few nm, the particle Reynolds number is rarely more than $10^{-3}$. In this so-called Stokes regime viscous forces dominate and inertial effects can be completely ignored. The Navier-Stokes equations can be replaced by the linear Stokes equations [57] so that analytic solutions are easier to obtain [63]. However, some of the resulting behavior is nonintuitive for those used to hydrodynamic effects on a macroscopic scale. For example, as famously explained by Purcell [77], many simple processes in biology occur on small length scales, well into the Stokes regime. The conditions that bacteria, typically a few $\mu \mathrm{m}$ long, experience in water are more akin to those humans would experience in extremely thick molasses. Similarly, colloids, polymers, vesicles, and other small suspended objects are all subject to the same physics, their motion dominated by viscous forces. For example, if for a colloid sedimenting at $1 \mu \mathrm{m} / \mathrm{s}$, gravity were instantaneously turned off, then the Stokes equations suggest that it would come to a complete halt in a distance significantly less than $1 \AA$, reflecting the irrelevance of inertial forces in this lowReynolds-number regime. It should be kept in mind that when the Stokes regime is reached because of small length scales (as opposed to very large viscosities such as those found for volcanic lava flows), then thermal fluctuations are also important. These will drive diffusive behavior [78]. In many ways SRD is ideally suited for this regime of small particles because the thermal fluctuations are naturally included [49].

\section{Dependence of the Reynolds number on simulation parameters}

From Eqs. (28) and (29), it follows that the Reynolds number for a colloid of hydrodynamic radius $a \approx \sigma_{c f}$ can be written as 


$$
\operatorname{Re}=\sqrt{\frac{5}{3}} \operatorname{Ma}\left(\frac{\sigma_{c f}}{a_{0}}\right)\left(\frac{\nu_{0}}{\nu}\right) .
$$

Equating the hydrodynamic radius $a$ to $\sigma_{c f}$ from the fluidcolloid WCA interaction of Eq. (17) is not quite correct, as we will see in Sec. V, but is a good enough approximation in light of the fact that these hydrodynamic numbers are qualitative rather than quantitative indicators.

In order to keep the Reynolds number low, one must either use small particles, very viscous fluids, or low velocities $v_{s}$. The latter condition is commensurate with a low Mach number, which is also desirable. For small enough $\lambda$ [and ignoring for simplicity the factor $f_{\text {col }}^{\nu}(\gamma, \alpha)$ in Eq. (10)] the Reynolds number then scales as

$$
\mathrm{Re} \approx 23 \mathrm{Ma} \frac{\sigma_{c f}}{a_{0}} \lambda .
$$

Again, we see that a smaller mean free path $\lambda$, which enhances the collisional viscosity, also helps bring the Reynolds number down. This parameter choice is also consistent with a larger Schmidt number. Thus larger Schmidt and smaller Mach numbers both help keep the Reynolds number low for SRD. In principle a large viscosity can also be obtained for large $\lambda$, which enhances the kinetic viscosity, but this choice also lowers the Schmidt number and raises the Knudsen number, which, as we will see in the next subsection, is not desirable.

Just as was found for the Mach number, it is relatively speaking more expensive computationally to simulate for low Reynolds numbers because the flow velocity must be kept low, which means longer simulation times are necessary to reach time scales where the suspended particles or fluid flows have moved a significant distance. We therefore compromise and normally keep $\operatorname{Re} \leq 0.2$, which is similar to the choice made for LB simulations [14,79]. For many situations related to the flow of colloids, this should be sufficiently stringent.

\section{Knudsen number}

The Knudsen number Kn measures rarefaction effects and can be written as

$$
\mathrm{Kn}=\frac{\lambda_{\text {free }}}{a},
$$

where $a$ is a characteristic length scale of the fluid flow and $\lambda_{\text {free }}$ is the mean free path of the fluid or gas. For a colloid in SRD one could take $a \approx \sigma_{c f}$ and $\lambda_{\text {free }} \approx \lambda a_{0}$. For large Knudsen numbers $\mathrm{Kn} \gtrsim 10$ continuum Navier-Stokes equations completely break down, but even for much smaller Knudsen numbers, significant rarefaction effects are seen. For example, for flow in a pipe, where $a$ in Eq. (34) is taken to be the pipe radius, important noncontinuum effects are seen when $\mathrm{Kn} \gtrsim 0.1$. In fact it is exactly for these conditions of modest Knudsen numbers, when corrections to the NavierStokes become noticeable, that DSMC approaches are often used [36,37]. SRD, which is related to DSMC, may also be expected to work well for such flows. It could find important applications in microfluidics and other micromechanical devices where Knudsen effects are expected to play a role $[80,81]$.

Colloidal dispersions normally have very small Knudsen numbers because the mean free path of most liquid solvents is very small. For water at standard temperature and pressure $\lambda_{\text {free }} \approx 3 \AA$. Just as found for the other dimensionless numbers, coarse-graining typically leads to larger Knudsen numbers because of the increase of the mean free path. Making the Knudsen number smaller also typically increases the computational cost because a larger number of collisions need to be calculated. In our simulations, we keep $\mathrm{Kn}$ $\leqslant 0.05$ for spheres. This rough criterion is based on the observation that for small Knudsen numbers the friction coefficient on a sphere is expected to be decreased by a factor $1-\alpha \mathrm{Kn}$, where $\alpha$ is a material-dependent constant of order 1 [63], so that we expect Knudsen number effects to be of the same order as other coarse-graining errors. There are two ways to achieve small Knudsen numbers: one is by increasing $\sigma_{c f} / a_{0}$, and the other is by decreasing $\lambda$. The second condition is commensurate with a large Schmidt number or a small Reynolds number.

In Ref. [53], the Knudsen numbers for colloids were Kn $\approx 0.5$ and $\mathrm{Kn} \approx 0.8$, depending on their colloid-solvent coupling. Such large Knudsen numbers should have a significant effect on particle friction coefficients, and this may explain why these authors find that volume fraction $\phi$ has less of an effect on the sedimentation velocity than we do [52].

\section{E. Peclet number}

The Peclet number Pe measures the relative strength of convective transport to diffusive transport. For example, for a colloid of radius $a$, traveling at an average velocity $v_{s}$, the Peclet number is defined as

$$
\mathrm{Pe}=\frac{v_{s} a}{D_{\mathrm{col}}},
$$

where $D_{\text {col }}$ is the colloid diffusion coefficient. Just as for the Reynolds number, the Peclet number can be interpreted as a ratio of a diffusive to a convective time scale, but now the former time scale is not for the diffusion of momentum but rather it is given by the colloid diffusion time

$$
\tau_{D}=\frac{\sigma_{c f}^{2}}{D_{\mathrm{col}}},
$$

which measures how long it takes for a colloid to diffuse over a distance $\sigma_{c f}$. We again take $a \approx \sigma_{c f}$ so that, using Eq. (30), the Peclet number can be written as

$$
\mathrm{Pe}=\frac{\tau_{D}}{t_{S}}
$$

If $\mathrm{Pe} \gg 1$, then the colloid moves convectively over a distance much larger than its radius $\sigma_{c f}$ in the time $\tau_{D}$ that it diffuses over that same distance. Brownian fluctuations are expected to be less important in this regime. For $\mathrm{Pe} \ll 1$, on the other hand, the opposite is the case, and the main transport mechanism is diffusive [note that on long enough time 
scales $\left(t>\tau_{D} / \mathrm{Pe}^{2}\right)$ convection will always eventually "outrun" diffusion [78]]. It is sometimes thought that for low Peclet numbers hydrodynamic effects can be safely ignored, but this is not always true. For example, we found that the reduction of average sedimentation velocity with particle volume fraction, famously first explained by Batchelor [82], is independent of $\mathrm{Pe}$ number down to $\mathrm{Pe}=0.1$ at least [52].

\section{Dependence of the Peclet number on simulation parameters}

The highest Peclet number achievable in simulation is limited by the constraints on the Mach and Reynolds numbers. For example the Mach number sets an upper limit on the maximum Peclet number by limiting $v_{s}$. From Eqs. (29) and (35), it follows that the Peclet number can be rewritten in terms of the Reynolds number as

$$
\mathrm{Pe}=\frac{\nu}{D_{\mathrm{col}}} \operatorname{Re} \approx 6 \pi \gamma\left(\frac{\nu}{\nu_{0}}\right)^{2}\left(\frac{\sigma_{c f}}{a_{0}}\right) \operatorname{Re},
$$

where we have approximated $D_{\text {col }} \approx k_{B} T /\left(6 \pi \eta \sigma_{c f}\right)$. This shows that for a given constraint on the Reynolds number, increasing $\gamma$ or $\sigma_{c f}$ increases the range of accessible Peclet numbers. Similarly, when the kinematic viscosity is dominated by the collisional contribution, decreasing the dimensionless mean free path $\lambda$ will also increase the maximum Peclet number allowed since $\mathrm{Pe}^{\max } \sim \lambda^{-2} \mathrm{Re} \sim \lambda^{-1} \mathrm{Ma}$. However, these changes increase the computational cost of the simulation.

\section{FINITE-SIZE, DISCRETIZATION, AND INERTIAL EFFECTS}

The cost of an SRD simulation scales almost linearly with the number of fluid particles, $N_{f}$, in the system, and this contribution is usually much larger than the cost of including the colloidal degrees of freedom. To optimize the efficiency of a simulation, one would therefore like to keep $N_{f}$ as small as possible. This objective can be achieved by keeping $\sigma_{c f} / a_{0}$ and the box size $L$ small. Unfortunately both these choices are constrained by the errors they introduce. Reducing $\sigma_{c f} / a_{0}$ means that short-range hydrodynamic fields become less accurately resolved due to Knudsen number and discretization effects. Decreasing the box size $L$ falls foul of the long-range nature of the HI's, and therefore, just as for Coulomb interactions [62], finite-size effects such as those induced by periodic images must be treated with special care.

Increasing the flow velocity may also be desirable since more Stokes times $\tau_{S}=\sigma_{c f} / v_{s}$ can be achieved for the same number of SRD collision steps, thus increasing computational efficiency. The Mach number gives one constraint on $v_{s}$, but usually the more stringent constraint comes from keeping the Reynolds number low to prevent unwanted inertial effects.

\section{A. Finite-size effects}

\section{Finite-size correction to the friction}

The friction coefficient $\xi$ can be extracted from the Stokes $\operatorname{drag} \mathbf{F}_{d}$ on a fixed colloid in fluid flow:

$$
\mathbf{F}_{d}=-\xi \mathbf{v}_{\infty} \equiv-4 \pi \eta a \mathbf{v}_{\infty},
$$

where $\mathbf{v}_{\infty}$ is the flow field at large distances. The prefactor of 4 comes from using slip boundary conditions; it would be 6 for stick boundary conditions, as verified in [58]. In principle, since $\eta$ is accurately known from theory for SRD, this expression can be used to extract the hydrodynamic radius $a$, which is not necessarily the same as $\sigma_{c f}$, from a simulation, as we did in [52].

The hydrodynamic radius $a$ can also be directly calculated from theory. To derive this, it is important to recognize that there are two sources of friction [49]. The first comes from the local Brownian collisions with the small particles and can be calculated by a simplified Enskog-Boltzmann-type kinetic theory [83]:

$$
\xi_{E}=\frac{8}{3}\left(\frac{2 \pi k_{B} T M_{c} m_{f}}{M_{c}+m_{f}}\right)^{1 / 2} n_{f} \sigma_{c f}^{2},
$$

which is here adapted for slip boundary conditions. A related expression for stick boundary conditions, including that for rotational frictions, is described in [58], where it was shown that the short-time exponential decays of the linear and angular velocity autocorrelation functions are quantitatively described by Enskog theory.

The second contribution to the friction, $\xi_{S}$, comes from integrating the Stokes solution to the hydrodynamic field over the surface of the particle, defined here as $r=\sigma_{c f}$. These two contributions to the friction should be added in parallel to obtain the total friction $[83,84]$ (see also Appendix B):

$$
\frac{1}{\xi}=\frac{1}{\xi_{S}}+\frac{1}{\xi_{E}} .
$$

In contrast to the Enskog friction, which is local, we expect substantial box-size effects on the Stokes friction $\xi_{S}$ since it depends on long-range hydrodynamic effects. These can be expressed in terms of a correction factor $f\left(\sigma_{c f} / L\right)$ that should go to 1 for very large systems:

$$
\xi_{S}=4 \pi \eta \sigma_{c f} f^{-1}\left(\sigma_{c f} / L\right) .
$$

To measure this correction factor we plot, in Fig. 5, the form $4 \pi \eta \sigma_{c f}\left(1 / \xi-1 / \xi_{E}\right)$ for various system box sizes for which we have measured $\xi$ from the simulation and estimated $\xi_{E}$ from Eq. (40). As expected, the correction factor tends to 1 for smaller $\sigma_{c f} / L$. More detailed calculations [85,86], taking into account the effect of periodic boundaries, suggests that to lowest order in $\sigma_{c f} / L$ the correction factor should scale as

$$
f\left(\sigma_{c f} / L\right) \approx 1+2.837 \frac{\sigma_{c f}}{L} .
$$

Indeed, a least-squares fit of the data to this form gives a slope of $c \approx 2.9$, close to the theoretical value and in agreement with similar lattice Boltzmann simulations of a single colloidal sphere [15].

With these ingredients in hand, we can calculate the theoretical expected friction from Eqs. (40)-(43). We know from previous work that the Enskog contribution at short times and the hydrodynamic contribution at long times quantitatively reproduce the rotational and translational velocity 


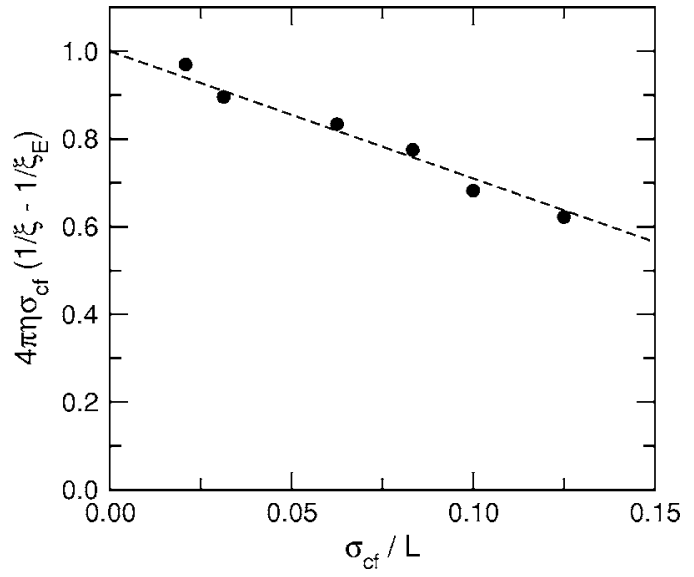

FIG. 5. Finite-system-size scaling of the Stokes friction $\xi_{S}$ on a sphere of size $\sigma_{c f}=2$ in a flow of velocity $v_{s}=0.01 a_{0} / t_{0}$. The correction factor $f$ defined in Eq. (42) can be extracted from measured friction $\xi$ (drag force/velocity) and estimated Enskog friction $\xi_{E}$ defined in Eq. (40). It compares well to theory, Eq. (43).

autocorrelation functions (VACF) for $\sigma_{c f} / a_{0}=2-5$ [58], and so we expect that the friction coefficients should also be accurately described by these theories. We test this further in Fig. 6 for a number of different values of $\sigma_{c f} / a_{0}$ and find excellent agreement with theory for $\operatorname{Re} \leqslant 1$ and $\sigma_{c f} / a_{0} \geqslant 2$. For $\sigma_{c f} / a_{0}=1$ and below, on the other hand, we find deviations from the theory. These are most likely due to Kn number and discretization effects, to be discussed in the next subsection.

For the smallest spheres the mass ratio $M_{c} / m_{f}$ may also change the measured friction if instead of fixing the sphere we were to let it move freely. For $\sigma_{c f}=a_{0}, M_{c} / m_{f} \approx 21$, which is small enough to have a significant effect [87]. For larger spheres, this is not expected to be a problem. For example, $M_{c} / m_{f} \approx 168$ for $\sigma_{c f}=2 a_{0}$ and $M_{c} / m_{f} \approx 1340$ for $\sigma_{c f}=4 a_{0}$.

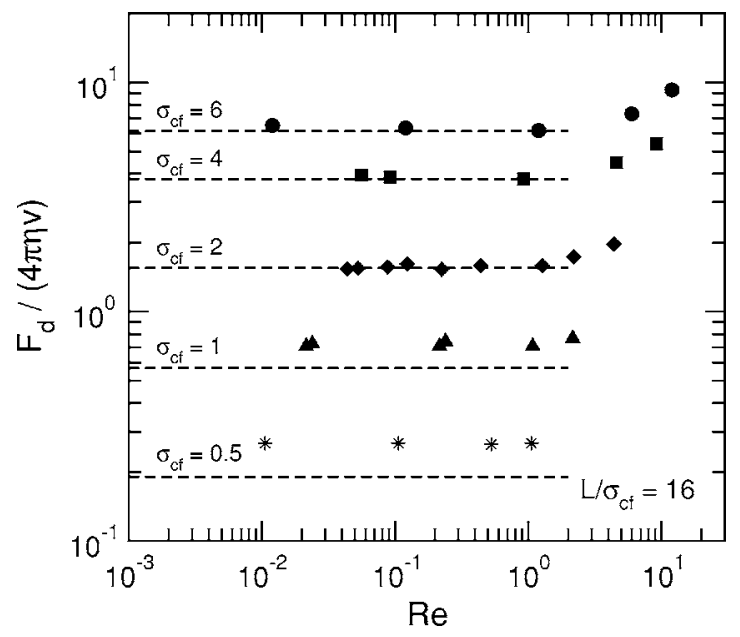

FIG. 6. Drag force $F_{d}$ divided by $4 \pi \eta v$ for various colloid sizes and Reynolds numbers (in all cases the box size $L=16 \sigma_{c f}$ ). For small Reynolds numbers this ratio converges to the effective hydrodynamic radius $a$. Dashed lines are theoretical predictions from combining Stokes and Enskog frictions in Eq. (41).

\section{Effective hydrodynamic radius}

From the calculated or measured friction we can also obtain the effective hydrodynamic radius $a_{\text {eff }}$, which is continuum concept, from a comparison of the microscopic frictions from Eq. (41) with Eq. (39):

$$
a_{\mathrm{eff}}=\frac{\xi_{S} \xi_{E}}{4 \pi \eta\left(\xi_{S}+\xi_{E}\right)}
$$

(for stick boundaries the $4 \pi$ should be replaced by $6 \pi$ ). We find $a_{\mathrm{eff}}\left(\sigma_{c f}=6\right)=6.11 a_{0}, a_{\mathrm{eff}}\left(\sigma_{c f}=4\right)=3.78 a_{0}$, and $a_{\mathrm{eff}}\left(\sigma_{c f}\right.$ $=2)=1.55 a_{0}$. The effective hydrodynamic radius is increased by the finite-size effects, but lowered by the Enskog contribution which is added in parallel. Because this latter contribution is relatively more important for small colloids, $a_{\mathrm{eff}}$ $<\sigma_{c f}$ for smaller $\sigma_{c f}$. For $\sigma_{c f}=6 a_{0}$ the Enskog contribution is smaller than the finite-size effects, and so the effective hydrodynamic radius is larger than $\sigma_{c f}$. Obviously this latter effect depends on the box size. In an infinite box $a_{\mathrm{eff}}<\sigma_{c f}$ for all values of $\sigma_{c f}$, due to the Enskog contribution. Note that it is the effective hydrodynamic radius $a_{\text {eff }}$ which sets the long-range hydrodynamic fields, as we will see below.

\section{Turning off long-range hydrodynamics}

Hydrodynamic forces can be turned off in SRD by regularly randomizing the absolute fluid particle velocities (with, for example, a naive Langevin thermostat). For particles embedded in an SRD solvent through participation in the collision step, this trick can be used to compare the effects of hydrodynamics to a purely Brownian simulation [40]. The Yeomans group has successfully applied this idea in a study of polymer collapse and protein folding [47].

However, for the case of the colloids embedded through direct solvent collisions, turning off the hydrodynamic forces by randomizing the velocities greatly enhances the friction because, as is clear from Eqs. (40)-(42), the two contributions add in parallel. Without long-range hydrodynamics, the friction would be entirely dominated by the Enskog contribution (40) which scales with $\sigma_{c f}^{2}$ and can be much larger than the hydrodynamic contribution which scales as $\sigma_{c f}$. Another way of stating this would be that by locally conserving momentum, SRD allows the development of long-range hydrodynamic fluid velocity correlations that greatly reduce the friction felt by a larger colloidal particle compared to the friction it would feel from a purely random Brownian heat bath at the same temperature and number density $n_{f}=\gamma / a_{0}^{3}$.

\section{B. Discretization effects on the flow field and friction}

For pure Stokes flow $(\mathrm{Re}=0)$, the velocity around a fixed slip boundary sphere of (effective) hydrodynamic radius $a$ can be exactly calculated:

$$
\mathbf{v}_{\mathrm{St}}(\mathbf{r})=\mathbf{v}_{\infty}\left(1-\frac{a}{2 r}\right)-\mathbf{v}_{\infty} \cdot \hat{\mathbf{r}} \hat{\mathbf{r}} \frac{a}{2 r},
$$

where $\mathbf{v}_{\infty}$ is the velocity field far away from the sphere and $\mathbf{r}$ the vector pointing from the center of the sphere to a position inside the fluid, with corresponding unit vector $\hat{\mathbf{r}}=\mathbf{r} / r$. 

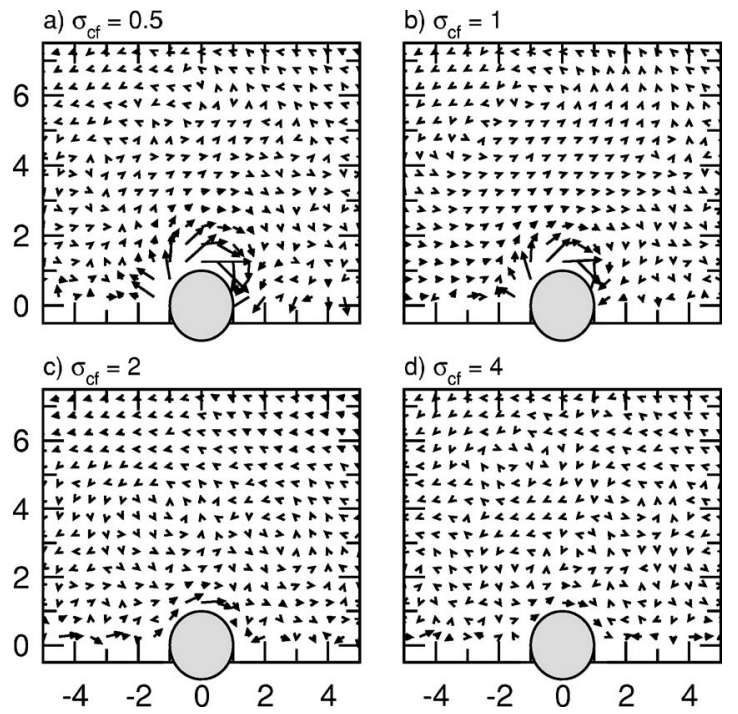

FIG. 7. Magnified difference fields $10\left[\mathbf{v}(\mathbf{r})-\mathbf{v}_{\mathrm{St}}(\mathbf{r})\right] / v_{\infty}$ for different colloidal sizes (the axes are scaled by $\sigma_{c f}$ ). In all cases Re $\approx 0.1$ and box size $L=16 \sigma_{c f}$.

In Fig. 7 we plot the difference between the measured field and the theoretical expected field (45) for four different colloid radius to cell size ratios $\sigma_{c f} / a_{0}$, using the values of the effective hydrodynamic radius $a$ calculated from combining Eqs. (41) and (39). As expected, the field is more accurately reproduced as the colloid radius becomes larger with respect to the cell size $a_{0}$. This improvement arises because SRD discretization and hydrodynamic Knudsen number effects become smaller. In Fig. 7 we observe quite large deviations in the hydrodynamic field for $\sigma_{c f} \leqslant 1$. These discretization effects may explain why the measured frictions in Fig. 6 do not agree with theory for these smallest sphere sizes. We also note that using $a=\sigma_{c f}$ instead of the more accurate value of the effective hydrodynamic radius $a$ calculated from theory results in significantly larger deviations between measured and theoretical flow fields. This independently confirms the values of the effective hydrodynamic radius.

The increased accuracy from using larger $\sigma_{c f} / a_{0}$ comes at a sharp increase in computational cost. To make sure that the finite-size effects in each simulation of Fig. 7 are approximately the same, the box size was scaled as $L / \sigma_{c f}$. This means that doubling the colloid size leads to an eightfold increase in the number of fluid particles. Moreover, the maximum velocity of the fluid must go down linearly in colloid size to keep the Reynolds number, defined in Eq. (29), constant. If in addition we keep the number of Stokes times fixed, meaning that the fluid flows a certain multiple of the colloid radius or the box size, then a larger particle also means the fluid needs to flow over a proportionally longer total distance. The overall computational costs for this calculation then scales at least as $\left(\sigma_{c f} / a_{0}\right)^{5}$, which is quite steep. For that reason, we advocate using smaller colloids wherever possible.

In most of our simulations we choose $\sigma_{c f}=2$, which leads to a small relative error in the full velocity field and for which we can fully explain the observed friction (as seen in the previous subsection). This size is similar to what is commonly used in LB [14,79].
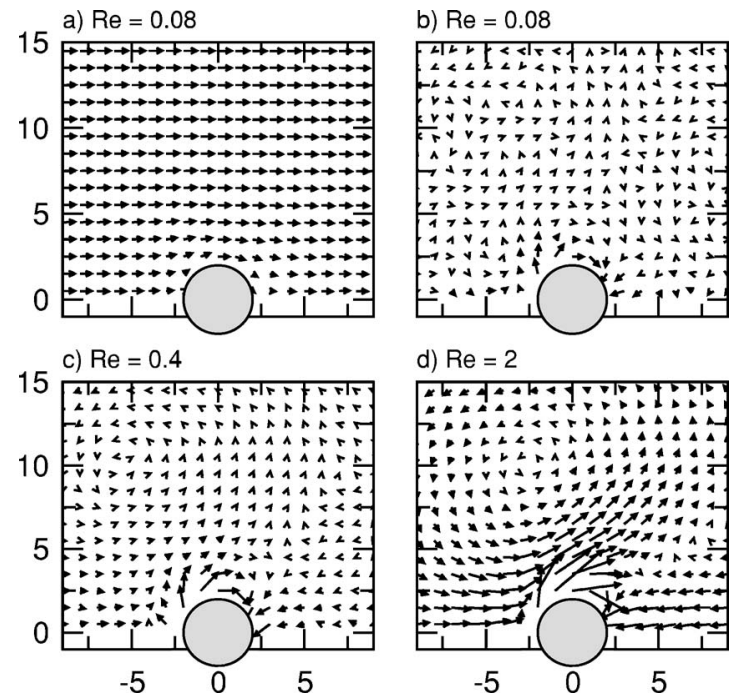

FIG. 8. (a) Fluid flow field around a fixed colloid with $\sigma_{c f}=2$ and $L=64 a_{0}$ for $\operatorname{Re}=0.08$. (b)-(d) Difference field $10[\mathbf{v}(\mathbf{r})$ $\left.-\mathbf{v}_{\mathrm{St}}(\mathbf{r})\right] / v_{\infty}$ for $\mathrm{Re}=0.08,0.4$, and 2, respectively.

\section{Inertial effects on the flow field and friction}

One of the challenges in SRD is to keep the Reynolds number down. It is virtually impossible to reach the extremely-low-Reynolds-number (Stokes) regime of realistic colloids, but that is not necessary either. In Fig. 6 we see that the friction only begins to noticeably vary from the Stokes limit at $\mathrm{Re} \approx 1$ (at least on a logarithmic scale). We expect the flow field itself to be more sensitive to finite-Reynoldsnumber effects. To study this directly, we examine, in Fig. 8, the flow field around a fixed colloid of size $\sigma_{c f}=2 a_{0}$ in a box of $L=32 a_{0}$ for for different values of $N_{\mathrm{Re}}$. The differences with the Stokes flow field of Eq. (45) are shown in Figs. $8(\mathrm{~b})-8(\mathrm{~d})$ for $\mathrm{Re}=0.08,0.4$, and 2 respectively. In all cases we used a hydrodynamic radius of $a=1.55$ for the theoretical comparison, as explained in the previous subsection. The lengths of the vectors in Figs. 8(b)-8(d) are multiplied by 10 for clarity. We observe that the relative errors increase with Re. They are on the order of $5 \%$ for $\mathrm{Re}=0.08$ and increase to something on the order of $40 \%$ for $\mathrm{Re}=2$. This is exactly what is expected, of course, as we are moving away from the Stokes regime with which these flow lines are being compared. Since we also expect effects on the order of a few $\%$ from the Knudsen number, Mach number, and various finitesize effects, we argue that keeping $\operatorname{Re} \leqslant 0.2$ should be good enough for most of the applications we have in mind.

\section{HIERARCHY OF TIME SCALES}

\section{A. Colloidal time scales}

Many different time scales govern the physics of a colloid of mass $M_{c}$ embedded in a solvent [1]. The most important ones are summarized in Table III and discussed in more detail below.

\section{Fluid time scales}

The shortest of these is the solvent collision time $\tau_{\text {col }}$ over which fluid molecules interact with each other. For a typical 
TABLE III. Time scales relevant for colloidal suspensions.

Solvent time scales

Solvent collision time over which solvent molecules

interact

Solvent relaxation time over which solvent velocity correlations decay

Hydrodynamic time scales

Sonic time over which sound propagates one colloidal radius

Kinematic time over which momentum (velocity) diffuses one colloidal radius

Stokes time over which a colloid converts over its own radius

Brownian time scales

Fokker-Planck time over which force-force correlations decay

Enskog relaxation time over which short-time colloid velocity correlations decay

Brownian relaxation time over which colloid velocity correlations decay in the Langevin equation

Colloid diffusion time over which a colloid diffuses over its radius

Ordering of time scales for colloidal particles

$\tau_{\mathrm{col}}<t_{f}, \tau_{F P}<\tau_{E}, \tau_{c S}<\tau_{B}<\tau_{\nu}<\tau_{D}, t_{S}$

$$
\begin{aligned}
& \tau_{\mathrm{col}} \approx 10^{-15} \mathrm{~s} \\
& \tau_{f} \approx 10^{-14}-10^{-13} \mathrm{~s} \\
& t_{c s}=\frac{a}{c_{s}} \\
& \tau_{\nu}=\frac{a^{2}}{\nu} \\
& t_{S}=\frac{a}{v_{s}}=\frac{\tau_{D}}{\mathrm{Pe}} \\
& \tau_{F P} \\
& \tau_{E}=\frac{M_{c}}{\xi_{E}} \\
& \tau_{B}=\frac{M_{c}}{\xi_{S}} \\
& \tau_{D}=\frac{a^{2}}{D_{\mathrm{col}}}
\end{aligned}
$$

molecular fluid, $\tau_{\text {col }}$ is on the order of a few tens of fs and any MD scheme for a molecular fluid must use a discretization time step $t_{M D} \ll \tau_{\text {col }}$ to properly integrate the equations of motion.

The next time scale up is the solvent relaxation time $\tau_{f}$, which measures how fast the solvent VACF decays. For a typical molecular liquid, $\tau_{f} \approx 10^{-14}-10^{-13} \mathrm{~s}$ (for water, at room temperature, for example, it is on the order of $50 \mathrm{fs}$ ).

\section{Hydrodynamic time scales for colloids}

Hydrodynamic interactions propagate by momentum (vorticity) diffusion and also by sound. The sonic time

$$
t_{c s}=\frac{a}{c_{s}}
$$

it takes a sound wave to travel the radius of a colloid is typically very small, on the order of $1 \mathrm{~ns}$ for a colloid of radius $a=1 \mu \mathrm{m}$. For that reason, sound effects are often ignored for colloidal suspensions under the assumption that they will have dissipated so quickly that they have no noticeably influence on the dynamics. However, some experiments [88] and theory [89] do find effects from sound waves on colloidal hydrodynamics, so that this issue is not completely settled yet.

The kinematic time $\tau_{\nu}$, defined in Eq. (31) (and Table III) as the time for momentum (vorticity) to diffuse over the radius of a colloid, is particularly important for hydrodynamics. It sets the time scale over which hydrodynamic interactions develop. For a colloid of radius $1 \mu \mathrm{m}, \tau_{\nu} \approx 10^{-6} \mathrm{~s}$, which is much faster the colloidal diffusion time $\tau_{D}$.

When studying problems with a finite flow velocity, another hydrodynamic time scale emerges. The Stokes time $t_{S}$, defined in Eq. (30) (and Table III) as the time for a colloid to advect over its own radius, can be related to the kinematic time by the relation $\tau_{\nu}=\operatorname{Re} t_{S}$. Because colloidal particles are in the Stokes regime where $\operatorname{Re} \ll 1$, we find $\tau_{\nu} \ll t_{S}$.

Simulation and analytical methods based on the Oseen tensor and its generalizations_-e.g., [4,5]—implicitly assume that the hydrodynamic interactions develop instantaneously-i.e., that $\tau_{\nu} \approx 0$. For the low-Reynoldsnumber regime of colloidal dispersions this is indeed a good approximation. Of course it must be kept in mind that $\tau_{\nu}$ is a diffusive time scale, so that the distance over which it propagates grows as $\sqrt{t}$. Thus the approximation of instantaneous hydrodynamics must be interpreted with some care for effects on larger length scales.

\section{Brownian time scales for colloids}

The fastest time scale relevant to the colloidal Brownian motion is the Fokker-Planck time $\tau_{F P}$ defined in [1] as the time over which the force-force correlation function decays. It is related to $\tau_{f}$, the time over which the fluid loses memory of its velocity because the forces on the colloid are caused by 
collisions with the fluid particles, velocity differences decorrelate on a time scale of order $\tau_{f}$.

The next time scale in this series is the Brownian time

$$
\tau_{B}=\frac{M_{c}}{\xi_{S}}
$$

where $\xi_{S}=6 \pi \eta a$ is the Stokes friction for stick boundary conditions. It is often claimed that this measures the time for a colloid to lose memory of its velocity. However, as discussed in Appendix B, this picture, based on the Langevin equation, is in fact incorrect for colloids. Nevertheless, $\tau_{B}$ has the advantage that it can easily be calculated and so we will use it as a crude upper bound on the colloid velocity decorrelation time.

Perhaps the most important time scale relevant for Brownian motion is the diffusion time $\tau_{D}$, described in Eq. (36) as the time for a colloidal particle to diffuse over its radius. For a colloid of radius $1 \mu \mathrm{m}, \tau_{D} \approx 5 \mathrm{~s}$, and even though $\tau_{D} \sim a^{3}$, it remains much larger than most microscopic time scales in the mesoscopic colloidal regime.

\section{B. Time scales for coarse-grained simulation}

In the previous subsection we saw that the relevant time scales for a single colloid in a solvent can span as many as 15 orders of magnitude. Clearly it would be impossible to bridge all the time scales of a physical colloidal systemfrom the molecular $\tau_{\text {col }}$ to the mesoscopic $\tau_{D}$-in a single simulation. Thankfully, it is not necessary to exactly reproduce each of the different time scales in order to achieve a correct coarse-graining of colloidal dynamics. As long as they are clearly separated, the correct physics should still emerge.

Since we take the view that the "solvent" particles are really a Navier-Stokes solver with noise, what is needed to reproduce Brownian behavior is first of all that the colloid experiences random kicks from the solvent on a short enough time scale. By dramatically reducing the number of "molecules" in the solvent, the number of kicks per unit of time is similarly reduced. Here we identify the Fokker-Planck time $\tau_{F P}$ as the time scale on which the colloid experiences random Brownian motion. For Brownian motion it does not really matter how the kicks are produced - they could be completely uncorrelated-but since we also require that the solvent transports momentum, solvent particles must have some kind of correlation, which in our case is represented by the time $\tau_{f}$. Other colloid relaxation times should be much longer than this. We therefore require first of all that $\tau_{F P}$ $\ll \tau_{B}$ and $\tau_{f} \ll \tau_{B}$.

Second, the colloid's VACF should decay to zero well before it has diffused or convected over its own radius. A proper separation of time scales in a coarse-graining scheme then requires that $\tau_{B} \ll \tau_{D}$ as well as $\tau_{B} \ll t_{S}$ for systems where convection is important.

Finally, for the correct hydrodynamics to emerge we require that $\tau_{F P}, \tau_{f} \ll \tau_{\nu} \ll \tau_{D}$. But this separation no longer needs to be over many orders of magnitude. As argued in [52], one order of magnitude separation between time scales should be sufficient for most applications. We illustrate this approach in Fig. 9.

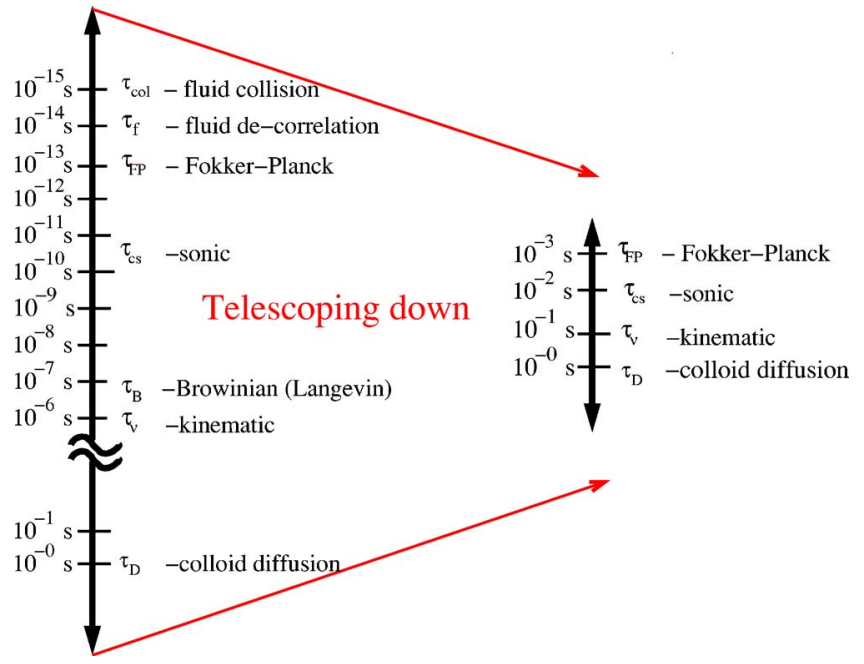

FIG. 9. (Color online) Schematic depiction of our strategy for coarse-graining across the hierarchy of time scales for a colloid (here the example taken is for a colloid of radius $1 \mu \mathrm{m}$ in $\mathrm{H}_{2} \mathrm{O}$ ). We first identify the relevant time scales and then telescope them down to a hierarchy which is compacted to maximize simulation efficiency, but sufficiently separated to correctly resolve the underlying physical behavior.

In the next few subsections we discuss in more quantitative detail how our coarse-graining strategy telescopes down the hierarchy of time scales in order to maximize the efficiency of a simulation while still retaining key physical features.

\section{SRD fluid time scales}

In SRD the physical time scale $\tau_{\text {col }}$ is coarse-grained out and the effect of the collisions calculated in an average way every time step $\Delta t_{c}$. The time scale $\tau_{f}$ on which the velocity correlations decay can be quite easily calculated from a random-collision approximation. Following [56], $\tau_{f} \approx-\lambda t_{0} / \ln \left[1-\frac{2}{3}(1-\cos \alpha)(1-1 / \gamma)\right]$. For our parameters $\alpha=\frac{1}{2} \pi$ and $\gamma=5$, we find $\tau_{f} \approx 0.76 \Delta t_{c}=0.076 t_{0}$. However, it should be kept in mind that for small $\lambda$ the exponential decay with $t / \tau_{f}$ turns over to a slower algebraic decay at larger $t$ [56]. The amplitude of this "tail" is, however, quite small.

\section{Hydrodynamic time scales for simulation}

For our choice of units $c_{s}=\sqrt{5 / 3} a_{0} / t_{0}$, so that the sonic time of Eq. (46) reduces to

$$
t_{c s} \approx 0.775 \frac{\sigma_{c f}}{a_{0}},
$$

which is independent of $\lambda$ or $\gamma$.

In the limit of small $\lambda$, the ratio of the kinematic time $\tau_{\nu}$ to $\Delta t_{c}$ can be simplified to the following form:

$$
\frac{\tau_{\nu}}{\Delta t_{c}} \approx 18 \frac{\sigma_{c f}^{2}}{a_{0}^{2}},
$$

so that the condition $\tau_{f}, \tau_{F P} \ll \tau_{\nu}$ is very easy to fulfill. Furthermore, under the same approximations, the ratio $\tau_{\nu} / t_{c s}$ 


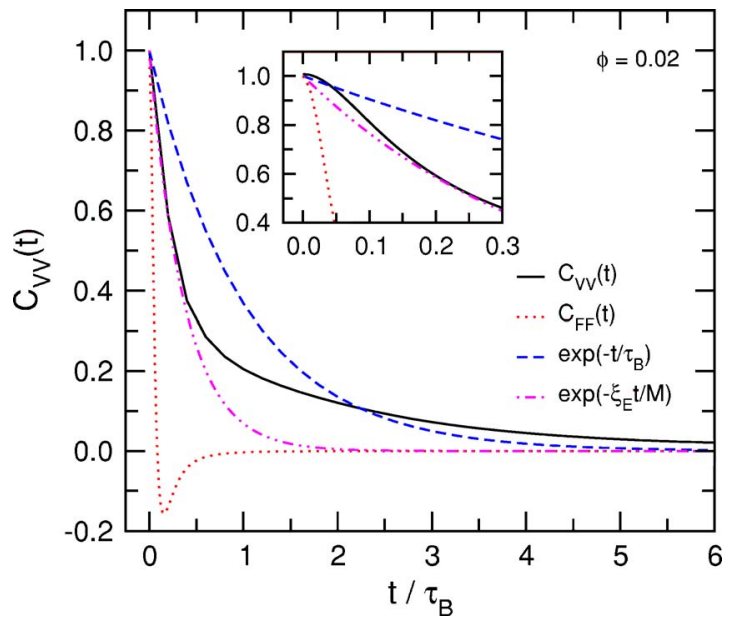

FIG. 10. (Color online) Normalized VACF for a single colloid with $\sigma_{c f}=2 a_{0}$ and $L=32 a_{0}$ : measured (solid line), Enskog shorttime prediction (dot-dashed line), and Brownian approximation (dashed line). The normalized force-force auto correlation $C_{F F}(t)$ (dotted line) decays on a much faster time scale. The inset shows a magnification of the short-time regime. Note that the colloid exhibits ballistic motion on a time scale $\sim \tau_{f}$. The time scales $\tau_{F P}$ $\approx 0.09, \tau_{B} \approx 2.5$, and $\tau_{D} \approx 200$ are clearly separated by at least an order of magnitude, as required.

$\approx 23 \sigma_{c f} \lambda$ so that for $\lambda$ too small the kinematic time becomes faster than the sonic time. For the simulation parameters used in [52] (and in Fig. 10), we find $\tau_{\nu} / \tau_{c s} \approx 5$.

\section{Brownian time scales for simulation}

We expect the Fokker-Planck time $\tau_{F P}$ to scale as $\Delta t_{c}$, since this is roughly equivalent to the time $\tau_{f}$ over which the fluid velocities will have randomized. In Fig. 10 we plot the force-force correlation function

$$
C_{F F}(t)=\frac{\langle F(t) F(0)\rangle}{\left\langle F^{2}\right\rangle} .
$$

Its short-time behavior is dominated by the random forces, and the initial decay time gives a good estimate of $\tau_{F P}$. As can be seen in the inset of Fig. 10, $\tau_{F P} \approx 0.09 t_{0}$, which is indeed on the order of $\Delta t_{c}$ or $\tau_{f}$.

If we make the reasonable approximation that $\sigma_{c c} \approx 2 \sigma_{c f}$, then the ratio of the Brownian time $\tau_{B}$ to the kinematic time $\tau_{\nu}$ simplifies to

$$
\frac{\tau_{B}}{\tau_{\nu}} \approx \frac{2 \rho_{c}}{9 \rho_{f}},
$$

so that the ratio of $\tau_{B}$ to $\tau_{\nu}$ is the same as for a real physical system. Since buoyancy requirements mean that normally $\rho_{c} \approx \rho_{f}$, the time scales are ordered as $\tau_{B}<\tau_{\nu}$. Moreover, since the ratio $\tau_{\nu} / \Delta t_{c} \gg 1$, independently of $\gamma$ or even $\lambda$ (as long as $\lambda \ll 1$ ), the condition that $\tau_{F P} \ll \tau_{B}$ is also not hard to fulfill in an SRD simulation.

These time scales are illustrated in Fig. 10 where we plot the normalized time correlation function

$$
C_{V V}(t)=\frac{\langle V(t) V(0)\rangle}{\left\langle V^{2}\right\rangle},
$$

with $V$ the Cartesian velocity coordinate of a single colloid. After the initial non-Markovian quadratic decay, the subsequent short-time exponential decay is not given by $\tau_{B}$ but instead by the Enskog time

$$
\tau_{E}=\frac{M_{c}}{\xi_{E}},
$$

where $\xi_{E}$ is the Enskog friction which can be calculated from kinetic theory $[58,83]$ [see Eq. (40)] and generally $\tau_{E}<\tau_{B}$. The physical origins of this behavior are described in more detail in Appendix B.

The colloid diffusion coefficient is directly related to the friction by the Stokes-Einstein relation

$$
D_{\mathrm{col}}=\frac{k_{B} T}{\xi} .
$$

If we assume that $\lambda \ll 1$ and, for simplicity, that $\xi \approx \xi_{S}$-i.e., we ignore the Enskog contribution-then the diffusion time scales as

$$
\tau_{D}=\frac{\sigma_{c f}^{2}}{D_{\mathrm{col}}} \approx \frac{6 \pi \eta \sigma_{c f}^{3}}{k_{B} T} \approx \frac{\gamma}{\lambda}\left(\frac{\sigma_{c f}}{a_{0}}\right)^{3} t_{0} \approx \frac{\gamma}{4 \lambda^{2}}\left(\frac{\sigma_{c f}}{a_{0}}\right) \tau_{B},
$$

so that $\tau_{B} \ll \tau_{D}$ is not hard to fulfill.

It is also instructive to examine the ratio of the diffusion time $\tau_{D}$ to the kinematic time $\tau_{\nu}$ :

$$
\frac{\tau_{D}}{\tau_{\nu}}=\frac{\nu}{D_{\mathrm{col}}} \approx 0.06 \frac{\gamma}{\lambda^{2}}\left(\frac{\sigma_{c f}}{a_{0}}\right) .
$$

In general we advocate keeping $\lambda$ small to increase the Schmidt number $\mathrm{Sc}=\nu / D_{f}$, and since another obvious constraint is $D_{\text {col }} \ll D_{f}$, there is not too much difficulty achieving the desired separation of time scales $\tau_{\nu} \ll \tau_{D}$.

As a concrete example of how these time scales are separated in a simulation, consider the parameters used in Fig. 10 for which we find $\tau_{f}=0.076 t_{0}, \tau_{F P}=0.09 t_{0} \tau_{B}=2.5 t_{0}, \tau_{\nu}=8 t_{0}$, and $\tau_{D}=200 t_{0}$. But more generally, what the analysis of this section shows is that obtaining the correct hierarchy of time scales,

$$
\tau_{f}, \tau_{F P} \ll \tau_{E}, \tau_{B}<\tau_{\nu} \ll \tau_{D}, t_{S},
$$

is virtually guaranteed once the conditions on dimensionless numbers, detailed in Sec. IV, are fulfilled.

\section{Measurements of diffusion}

To further test the hybrid MD-SRD coarse-graining scheme, we plot in Fig. 11 the time-dependent diffusion coefficient $D(t)$, defined as

$$
D(t)=\int_{0}^{t} d t^{\prime}\left\langle V\left(t^{\prime}\right) V(0)\right\rangle,
$$

for a number of different volume fractions $\phi=\frac{4}{3} N / V \pi \sigma_{c f}^{3}$. Note that the convergence slows with increasing volume 


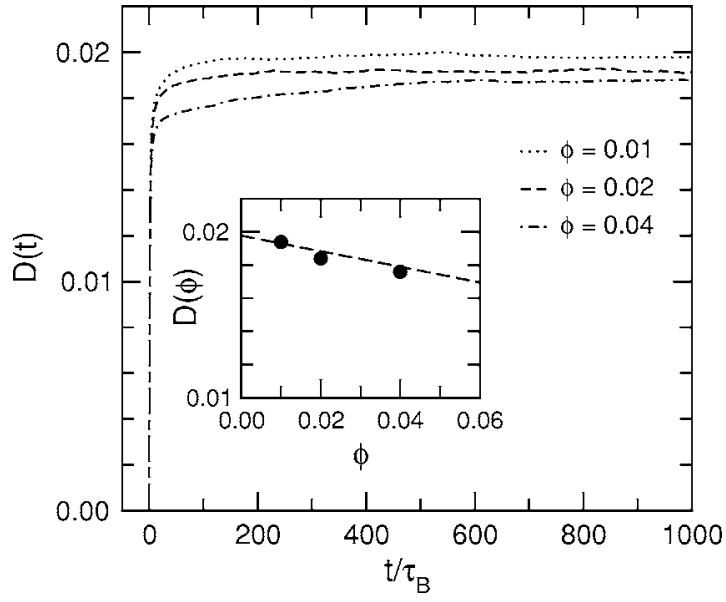

FIG. 11. Time-dependent self-diffusion coefficient $D(t)$ for different volume fractions $\phi$, achieved by varying the number of colloids in a box of fixed volume. The inset shows the self-diffusion coefficient $(D=\lim t \rightarrow \infty D(t))$ as a function of volume fraction, together with an analytical prediction $D=D_{0}(1-1.1795 \phi)$ [90] valid in the low-density limit.

fraction $\phi$. The infinite time integral gives the diffusion coefficient-i.e., $D_{\text {col }}=\lim _{t \rightarrow \infty} D(t)$. In the limit of low densities the diffusion coefficient has been predicted to take the form $D(\phi)=D_{0}(1-c \phi)$ with 1.1795 for slip boundary spheres [90] and this provides a good fit at the lower-volume fractions.

In Fig. 12 we plot the mean-square displacement of a Cartesian component of the colloidal particle position. As highlighted in the inset, at short times there is an initial ballistic regime due to motion at an average mean-square velocity $\left\langle V(0)^{2}\right\rangle=k_{B} T / M$, resulting in a mean-square displacement $\left\langle[X(t)-X(0)]^{2}\right\rangle=\left(k_{B} T / M\right) t^{2}$. At times $t \gg \tau_{\nu}$, the motion is clearly diffusive, as expected, with a linear dependence on $t$ and a slope of $2 D_{\text {col }}$, where $D_{\text {col }}$ is given by the infinite time limit of Eq. (58). On the scale of the plot the asymptotic

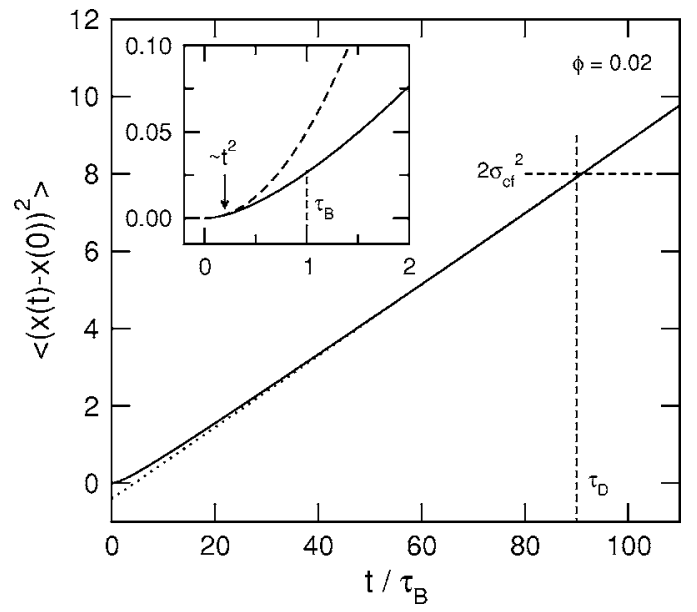

FIG. 12. Mean-square-displacement of a $\sigma_{c f}=2 a_{0}$ colloid (solid line). The dotted line is a fit to the long-time limit. The inset shows a magnification of the short-time regime, highlighting the initial ballistic regime [dashed line is $\langle V(0)\rangle^{2} t^{2}$, which rapidly turns over to the diffusive regime. regime is reached well before the time $\tau_{D}$ over which the particle has diffused, on average, over its radius. In Appendix B the behavior of $D(t)$ and the related mean-square displacement are discussed in more detail for time scales $t \ll \tau_{D}$.

\section{MAPPING BETWEEN A PHYSICAL AND COARSE-GRAINED SYSTEMS: NO FREE LUNCH?}

The ultimate goal of any coarse-graining procedure is to correctly describe physical phenomena in the natural world. As we have argued in this paper, it is impossible to bridge, in a single simulation, all the time and length scales relevant to a colloid suspended in a solvent. Compromises must be made. Nevertheless, a fruitful mapping from a coarsegrained simulation to a physical system is possible and greatly facilitated by expressing the physical properties of interest in dimensionless terms. The best way to illustrate this is with some examples.

\section{A. Example 1: Mapping to diffusive and Stokes time scales}

For many dynamic phenomena in colloidal dispersions, the most important time scale is the diffusion time $\tau_{D}$. To map a coarse-grained simulation onto a real physical system one could therefore equate the diffusion time and the colloid radius $a$ of the simulation to that of the real physical system. For example, take the simulation parameters from Table VI in Appendix $\mathrm{C}$ for $a=2 a_{0}$ and compare these to the properties of colloids of radius $a=1 \mu \mathrm{m}$ and $a=10 \mathrm{~nm}$ from Table $\mathrm{V}$. For the larger colloids, $\tau_{D}=5 \mathrm{~s}$ and equating this to the simulation $\tau_{D}$ means that $a_{0}=0.5 \mu \mathrm{m}$ and $t_{0}=0.02 \mathrm{~s}$. Performing the same exercise for the smaller colloid, where $\tau_{D}=5 \times 10^{-6} \mathrm{~s}$, results in $a_{0}=5 \mathrm{~nm}$ and $t_{0}=2 \times 10^{-8} \mathrm{~s}$. The first thing that becomes obvious from this exercise is that "physical" time is set here not by the coarse-grained simulation, but by the particular system that one is comparing to. In other words, many different physical systems could be mapped to the same simulation.

If the system is also subjected to flow, then a second time scale emerges, the Stokes time $t_{S}=\operatorname{Pe} \tau_{D}$. As long as the physical Peclet number is achievable without compromising the simulation quality, then this time scale can simultaneously be set equal to that of the physical system. Behavior that depends primarily on these two time scales should then be correctly rendered by the SRD hybrid MD scheme described in this paper.

\section{B. Example 2: Mapping to kinematic time scales}

By fixing $\tau_{D}$ or $t_{S}$, as in example 1 , other time scales are not correctly reproduced. For the large- and small-colloid systems described above, we would find $\tau_{\nu}=0.17 \mathrm{~s}$ and 1.7 $\times 10^{-7}$ s respectively, which contrasts with the physical values of $\tau_{\nu}=10^{-6} \mathrm{~s}$ and $10^{-10} \mathrm{~s}$ shown in Table $\mathrm{V}$. In other words, fixing $\tau_{D}$ results in values of $\tau_{\nu}$ that are too large by orders of magnitude. But of course these much larger values of $\tau_{\nu}$ are by design, because the SRD simulation is optimized by compacting the very large physical hierarchy of time scales. If we are interested in processes dominated by $\tau_{D}$, having different $\tau_{\nu}$ does not matter, as long as $\tau_{\nu} \ll \tau_{D}$. 
On the other hand, if we want to describe processes that occur on much smaller time scales-for example, long-time tails in colloidal VACF's - then simulation times $\tau_{\nu}$ could be mapped onto the physical $\tau_{\nu}$ instead. For the same $a=2 a_{0}$ simulation parameters used above, the $a=1 \mu \mathrm{m}$ system now maps onto $a_{0}=0.5 \mu \mathrm{m}$, and $t_{0}=1.3 \times 10^{-7} \mathrm{~s}$ with of course a strongly underestimated diffusion time: $\tau_{D}=3 \times 10^{-5} \mathrm{~s}$ instead of $5 \mathrm{~s}$. Similarly for the $a=10 \mathrm{~nm}$ system, the mapping is to $a_{0}=5 \mathrm{~nm}, t_{0}=1.3 \times 10^{-11} \mathrm{~s}$, and $\tau_{D}=3 \times 10^{-9} \mathrm{~s}$ instead of $5 \times 10^{-6} \mathrm{~s}$. For many processes on time scales $t<\tau_{D}$, this underestimate of $\tau_{D}$ does not really matter. It is only when time scales are mixed that extra care must be employed in interpreting the simulation results. And if the processes can be described in terms of different dimensionless times, then it should normally still be possible to disentangle the simulation results and correctly map onto a real physical system.

Another lesson from these examples of mapping to different time scales comes from comparing the kinematic viscosity of a physical system, which say takes the value $\nu$ $=10^{6} \mu \mathrm{m}^{2} / \mathrm{s}$ for water, to its value in SRD simulations. If for the $a=2 a_{0}$ SRD system described above we map time onto $\tau_{D}$, as in example 1 , then for the $a=1 \mu \mathrm{m}$ system $\nu$ $=6 \mu \mathrm{m}^{2} / \mathrm{s}$, while for the $a=10 \mathrm{~nm}$ system $\nu=6$ $\times 10^{-3} \mu \mathrm{m}^{2} / \mathrm{s}$. As expected, both are much smaller than the true value in water. If we instead map the times to $\tau_{\nu}$, then of course the kinematic viscosity takes the same value as for the physical system (since we also set the length scales equal to the physical length scales). This apparent ambiguity in defining a property like the kinematic viscosity is inherent in many coarse-graining schemes. We take the view that the precise value of $\nu$ is not important - the only thing that matters is that it is large enough to ensure a proper separation of times scales and the correct regime of hydrodynamic numbers.

\section{Example 3: Mapping mass and temperature}

In the examples above, we have only discussed setting lengths and times. This leaves either the mass $m_{f}$ or temperature (thermal energy) $k_{B} T$ still to be set. Because for many dynamic properties the absolute mass is effectively an irrelevant variable (although relative masses may not be), there is some freedom to choose. One possibility would be to set $M_{c}$, the mass of the colloid, equal to the physical mass. For an $a=1 \mu \mathrm{m}$ neutrally buoyant colloid this sets $m_{f}=2.5$ $\times 10^{-14} \mathrm{~g}$ (equal to about $8 \times 10^{8}$ water molecules) while for an $a=10 \mathrm{~nm}$ colloid we find $m_{f}=2.5 \times 10^{-20} \mathrm{~g}$ (equal to about 800 water molecules). By construction this procedure produces the correct physical $\rho_{f}$. If we fix time with $\tau_{\nu}$, this will therefore also generate the correct shear viscosity $\eta$. If instead we fix $\tau_{D}$, then $\eta$ will be much smaller than the physical value. For either choice of time scales, other properties, such as the fluid self-diffusion constant or the number density, will have different values from the underlying physical system.

Setting the mass in this way means that the unit of thermal energy $k_{B} T_{0}=m_{f} a_{0}^{2} t_{0}^{-2}$ would also be very large, leading to the appearance of very low temperatures. However, because temperature only determines behavior through the way it scales potentials, it is quite easy to simply renormalize the effective energy scales and obtain the same behavior as for the physical system. Alternatively one could set the temperature equal to that of a physical system, but that would mean that the masses would again differ significantly from the real physical system. At any rate, for the same simulation (say, of sedimentation at a given value of $\mathrm{Pe}$ ) the values of mass or temperature depend on the physical system one compares to. This apparent ambiguity (or flexibility) simply reflects the fact that the dominant effects of temperature and mass come into play as relative ratios and not as absolute values.

\section{Example 4: Mapping to attractive potentials-problems with length scales?}

So far we have only treated one length scale in the problem, the radius $a$. Implicitly we have therefore assumed that the colloid-colloid interaction does not have another intrinsic length scale of its own. For hard-sphere colloids this is strictly true, and for steep repulsions such as the WCA form of Eq. (16) it is also a fairly good assumption that the exact choice of the exponent $n$ in this equation does not significantly affect the dynamical properties [61]. Similarly, there is some freedom to set the repulsive fluid-colloid potential of Eq. (17) in such a way as to optimize the simulation parameters, in particular $\Delta t_{M D}$. The physical liquid-colloid interaction would obviously have a much more complex form, but SRD coarse-grains out such details. A similar argument can be made for colloid and fluid interactions with hard walls, needed, for example, to study problems with confinement, for which, inter alia, SRD is particularly well suited.

Things become more complicated for colloids with an explicit attractive interaction, such as DLVO or a depletion potential [2]. These potentials introduce a new length scale, the range of the attraction. The ratio of this length to the hard-core diameter helps determine the equilibrium properties of the fluid $[21,22]$. In a physical system this ratio may be quite small. Keeping the ratio the same in the simulations then leads to potentials that are very steep on the scale of $\sigma_{c c}$. The MD time step $\Delta t_{M D}$ may need to be very small in order to properly integrate the MD equations of motion. Such a small $\Delta t_{M D}$ can make a simulation very inefficient, as was found in [53] who followed this strategy and were forced to use $\Delta t_{M D} / \Delta t_{c} \approx 455$. However, in that case the DLVO potential dominates the behavior so that these simulations could be viewed as an MD simulation where the SRD was only included to roughly resolve the long-range hydrodynamics [91]. In general, we advocate adapting the potential to maximize simulation efficiency, while preserving key physical properties such as the topology of the phase diagram. For example, the attractive energy scale can be set by the dimensionless reduced second virial coefficient, which gives an excellent approximation for how far one is from the liquidliquid critical point [92]. When the attractive interaction range is less than about $30 \%$ of the colloid hard-core diameter, the colloidal "liquid" phase becomes metastable with respect to the fluid-solid phase transition. At low colloid packing fraction $\phi_{c}$ this leads to so-called "energetic fluid" behavior [21]. To simulate a colloidal suspension in this "en- 
ergetic fluid" regime, having a range of less than $30 \%$ of $a$ should suffice. In this way adding attractions does not have to make the simulation much less efficient. Moreover, the effects of these constraints, placed by the colloid-colloid interaction on $\Delta t_{M D}$, are to some degree mitigated by the fact that it is usually the colloid-fluid interaction which sets this time scale.

There are still a few final subtleties to mention. First of all, using small $\sigma_{c f} / a_{0}$ in the simulations may greatly increase efficiency, but it also leads to a relatively larger contribution of the Enskog friction to the total friction in Eq. (41). For physical colloids in a molecular solvent the exact magnitude of the Enskog friction is still an open question, but undoubtedly for larger colloids it is almost negligible. Some of the Enskog effects can simply be absorbed into an effective hydrodynamic radius $a$, but others must be interpreted with some care.

Another regime where we might expect to see deviations beyond a simple rescaling of time scales would be at very short times. For example, when colloids form a crystal, their oscillations can be decomposed into lattice phonons. All but the longest-wavelength longitudinal oscillations are overdamped due to the viscous drag and backflow effects of the solvent [93]. In SRD, however, some of the very shortwavelength oscillations might be preserved due to the fact that the motion on those time scales is still ballistic. These may have an impact on the interpretation of SRD simulations of microrheology.

In summary, these examples demonstrate that as long as the coarse-grained simulation produces the correct hydrodynamic and Brownian behavior, then there is considerable freedom in assigning real physical values to the parameters in the simulation. Attractive interactions may introduce a new length scale, but a judicious choice of a model potential should still reproduce the correct physical behavior. The same simulation may therefore be mapped onto multiple different physical systems. Conversely, for the same physical system, different choices can be made for the time scales that are mapped to physical values, but by design, not all time scales can be simultaneously resolved.

\section{CONCLUSION}

Correctly rendering the principal Brownian and hydrodynamic properties of colloids in solution is a challenging task for computer simulation. In this article we have explored how treating the solvent with SRD, which coarse-grains the collisions between solvent particles over both time and space, leads to an efficient solution of the thermohydrodynamic equations of the solvent, in the external field provided by the colloids. Although it is impossible to simulate the entire range of time scales encountered in real colloidal suspensions, which can span as much as 15 orders of magnitude, we argue that this is also not necessary. Instead, by recognizing first that hydrodynamic effects are governed by a set of dimensionless numbers and second that the full hierarchy of time scales can be telescoped down to a much more manageable range while still being properly physically separated, we demonstrate that the key Brownian and hydrody- namic properties of a colloidal suspension can indeed be captured by our SRD-based coarse-graining scheme.

In particular, to simulate in the colloidal regime, the modeler should ensure that the dimensionless hydrodynamic numbers such as the Mach, Reynolds, and Knudsen numbers are small enough $(\ll 1)$ and the Schmidt number is large enough $(\gg 1)$. While these numbers are constrained by approximate limits, the Peclet number, which measures the relative strength of the convective transport to the diffusive transport of the colloids, can be either larger or smaller than 1 , depending on physical properties such as the colloidal size and the strength of the external field that one wants to study. It turns out that if the hydrodynamic numbers above are chosen correctly, it is usually not so difficult to also satisfy the proper separation of the time scales.

We explored how to reach the desired regime of hydrodynamic numbers and time scales by tuning the simulation parameters. The two most important parameters are the dimensionless mean free path $\lambda$, which measures what fraction of a cell size $a_{0}$ an SRD particle travels between collisions performed at every time step $\Delta t_{c}$, and the ratio of the colloid radius $\sigma_{c f}$ to the SRD cell size $a_{0}$. The general picture that emerges is that the collision time $\Delta t_{c}$ must be chosen so that $\lambda$ is small since this helps keep the Schmidt number high and both the Knudsen and Reynolds numbers low. Of course, for computational efficiency, the collision time should not be too small either. Similarly, although a larger colloid radius means that the hydrodynamic fields are more accurately rendered, this should be tempered by the fact that the simulation efficiency drops off rapidly with increasing colloid radius. We find that choosing $\sigma_{c f} / a_{0} \approx 2$, which may seem small, already leads to accurate hydrodynamic properties.

A number of subtleties occur at the fluid-colloid interface, which may induce spurious depletion interactions between the colloids, but these can be avoided by a careful choice of potential parameters for slip boundary conditions. Stick boundary conditions can also be implemented, and we advocate using stochastic bounce-back rules to achieve this coarse-graining over the fluid-colloid interactions.

The simplicity of the SRD solvent facilitates the calculation of the the short-time behavior of the VACF from kinetic theory. We argue that for times shorter than the sonic time $t_{c s}=a / c_{s}$, where hydrodynamic collective modes will not yet have fully developed, the decay of the VACF is typically much more rapid than the prediction of the simple Langevin equation. The ensuing Enskog contribution to the total friction should be added in parallel to the microscopic hydrodynamic friction, and from the sum an analytic expression for the effective hydrodynamic radius $a_{\text {eff }}$ can be derived which is consistent with independent measurements of the longrange hydrodynamic fields.

Although we have shown how to correctly render the principal Brownian and hydrodynamic behavior of colloids in solution there is, as always, no such thing as a free lunch. The price to be paid is an inevitable consequence of compressing together the hierarchy of time and length scales: not all the simulation parameters can be simultaneously mapped onto a physical system of interest. However the cost of the "lunch" can be haggled down by recognizing that only a few physical parameters are usually important. For example, one 
could map the simulation onto real physical time-say, through $\tau_{D}$ and $t_{S}$ or, alternatively, through $\tau_{\nu}$; there is some freedom (or ambiguity) in the choice of which time scale to use. The correspondence with physical reality becomes more complex when different physical time scales mix, but in practice they can often be disentangled when both the coarse-grained simulation and the physical system are expressed in terms of dimensionless numbers and ratios. In other words, there is no substitute for careful physical insight which is, as always, priceless.

A number of lessons can be drawn from this exercise that may be relevant for other coarse-graining schemes. SRD is closely related to lattice Boltzmann dynamics and to the Lowe-Anderson thermostat, and many of the conclusions above should transfer in an obvious way. For dissipative particle dynamics, we argue that the physical interpretation is facilitated by recognizing that, just as for SRD, the DPD particles should not be viewed as "clumps of fluid" but rather as a convenient computational tool to solve the underlying thermo hydrodynamic equations. All these methods must correctly resolve the time-scale hierarchy and satisfy the relevant hydrodynamic numbers in order to reproduce the right underlying physics.

Our measurements of the VACF and the discussion in Appendix B show explicitly that Brownian dynamics simulations, which are based on the simple Langevin equation, do not correctly capture either the long- or short-time decay of colloidal VACF's.

A major advantage of SRD is the relative ease with which solutes and external boundary conditions (walls) are introduced. Boundaries may be hard or soft, with either stick or slip conditions. This method is therefore particularly promising for simulations in the fields of biofluidics and nanofluidics, where small mesoparticles flow in constrained geometries, possibly confined by soft fluctuating walls. We are currently exploring these possibilities.

\section{ACKNOWLEDGMENTS}

J.T.P. thanks the EPSRC and IMPACT Faraday, and A.A.L. thanks the Royal Society (London) for financial support. We thank W. Briels, H. Löwen, J. Lopez Lopez, J. Sané, A. Mayhew Seers, I. Pagonabarraga, A. Wilber, and A. Wysocki for very helpful conversations and W. den Otter for a careful reading of the manuscript.

\section{APPENDIX A: THERMOSTATTING OF SRD UNDER FLOW}

When an external field is applied to the fluid (or to objects embedded in the fluid), energy is pumped into the system and, as a consequence, the average temperature will rise. To prevent this from happening, the system must be coupled to a thermostat. In order not to influence the average flow, thermostatting requires a local and Galilean invariant definition of temperature.

We achieve this by relating the instantaneous local temperature in a cell to the mean-square deviation of the fluid particle velocities from the center-of-mass velocity of that cell. To minimize the interference of the thermostat with the dynamics, we choose to measure the overall temperature as

$$
\begin{gathered}
k_{B} T_{\text {meas }}=\frac{m_{f}}{N_{\text {free }}} \sum_{\text {cell } c} \sum_{c i \in c}\left(\mathbf{v}_{i}-\mathbf{v}_{\text {c.m. } . c}\right)^{2}, \\
N_{\text {free }}=\sum_{\text {cell } c} \begin{cases}3\left(\mathrm{~N}_{\text {cell } c}-1\right) & \left(N_{\text {cell } c}>1\right), \\
0 & \left(N_{\text {cell } c} \leqslant 1\right) .\end{cases}
\end{gathered}
$$

In Eq. (A2), $N_{\text {cell } c}$ is the instantaneous number of fluid particles within cell $c$. Three degrees of freedom must be subtracted for fixing the center-of-mass velocity of each cell (note that this implies that the local temperature is not defined in cells containing zero or one particle). During the simulations, the temperature $T_{\text {meas }}$ is measured every $\Delta t_{c}$ (this can be done very efficiently because the relative velocities are readily available in the collision step routine). The thermostat then acts by rescaling all relative velocities $\mathbf{v}_{i}$ $-\mathbf{v}_{\text {c.m., }}$ by a factor $\sqrt{T / T_{\text {meas }}}$. This strict enforcement of the overall temperature may be relaxed by allowing appropriate fluctuations, but in view of the very large number of fluid particles (typically $10^{6}$ ) this will not have a measurable effect on the dynamics of the fluid particles.

\section{APPENDIX B: THE LANGEVIN EQUATION AND MEMORY EFFECTS}

Within the Brownian approximation each Cartesian component of the colloid velocity $V$ is described by a simple Langevin equation of the form

$$
\begin{gathered}
M_{c} \frac{d V}{d t}=-\xi V+F^{R}(t), \\
\left\langle F^{R}(t) F^{R}\left(t^{\prime}\right)\right\rangle=2 k_{B} T \xi \delta\left(t-t^{\prime}\right),
\end{gathered}
$$

without any memory effect in the friction coefficient $\xi$ or in the random force $F^{R}(t)$. This Langevin equation fundamentally arises from the assumption that the force kicks are Markovian: each step is independent of any previous behavior. Of course on a very short time scale this is not true, but since Brownian motion is self-similar, the Markovian assumptions underlying Eq. (B1) might be expected to be accurate on longer time scales. Inspection of Fig. 10 shows that the force-force correlation function (50) does indeed decay on a time scale $\tau_{F P}$ which is much faster than other Brownian time scales in the system.

From the Langevin equation (B1) it follows that the velocity autocorrelation function takes the form

$$
\langle V(t) V(0)\rangle^{L}=\frac{k_{B} T}{M_{c}} \exp \left(-t / \tau_{B}\right),
$$

where $\tau_{B}=M_{c} / \xi$. The VACF is directly related to the diffusion constant through the Green-Kubo relation

$$
D_{\text {col }}=\lim _{t \rightarrow \infty} \int d t\langle V(t) V(0)\rangle=\frac{k_{B} T}{\xi},
$$

and Eq. (B3) has the attractive property that its integral gives the correct Stokes-Einstein form for $D_{\mathrm{col}}$ and that its $t=0$ 


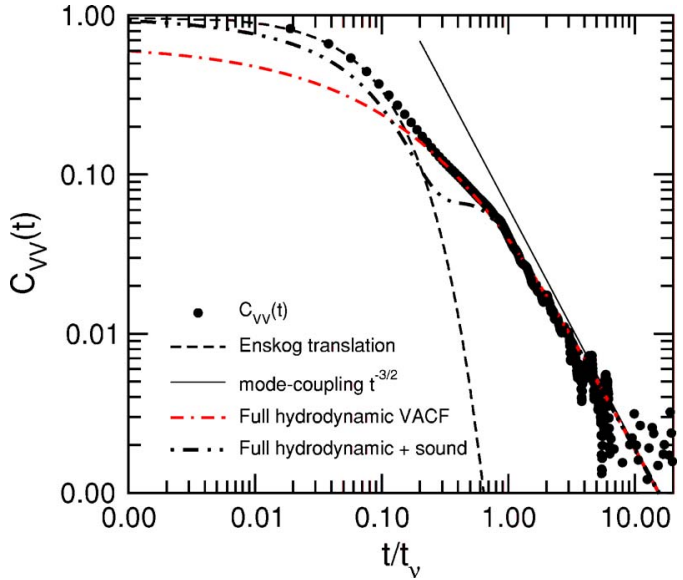

FIG. 13. (Color online) Normalized VACF for $a=2 a_{0}$ and stick boundary conditions (from [58]), compared to the short-time Enskog result (B11), and the full hydrodynamic VACF (B5) by itself, and with the VACF (B9) from sound added. We also show the asymptotic long-time hydrodynamic tail from mode-coupling theory.

limit gives the expected value from equipartition. It is therefore a popular pedagogical device for introducing Brownian motion. Notwithstanding its seductive simplicity, we will argue that this Langevin approach strongly underestimates the initial decay rate of the VACF and badly overestimates its long-time decay rate.

\section{VACF at long times}

At longer times, as first shown in MD simulations by Alder and Wainwright [94], the VACF shows an algebraic decay that is much slower than the exponential decay of Eq. (B3). The difference is due to the breakdown of the Markovian assumption for times $t \gg \tau_{F P}$. Memory effects arise because the momentum that a colloidal particle transfers to the fluid is conserved, with dynamics described by the NavierStokes equations, resulting in long-range flow patterns that affect a colloid on much longer time scales than Eq. (B2) suggests. Calculations taking into account the full timedependent hydrodynamics were performed by Hauge and Martin-Löf [95] and Hinch [96] (and others apparently much earlier [97]). For $\rho_{c}=\rho_{f}$-i.e., a neutrally buoyant particlethese expressions can be written as

$$
\langle V(t) V(0)\rangle^{H}=\frac{2}{3} \frac{k_{B} T}{M_{c}}\left(\frac{1}{3 \pi} \int_{0}^{\infty} d x \frac{x^{1 / 2} \exp \left[-x\left(t / \tau_{\nu}\right)\right]}{1+\frac{x}{3}+\frac{x^{2}}{9}}\right),
$$

where we have chosen to use an integral form as in [98]. Under the assumption that the sound effects have dissipated away, the only hydrodynamic time scale is the kinematic time $\tau_{\nu}$ defined in Eq. (31). The VACF must therefore scale with $t / \tau_{\nu}$, independently of colloid size. Equation (B5) is written in a way to emphasize this scaling.

By expanding the denominator it is not hard to see that at longer times the well-known algebraic tail results:

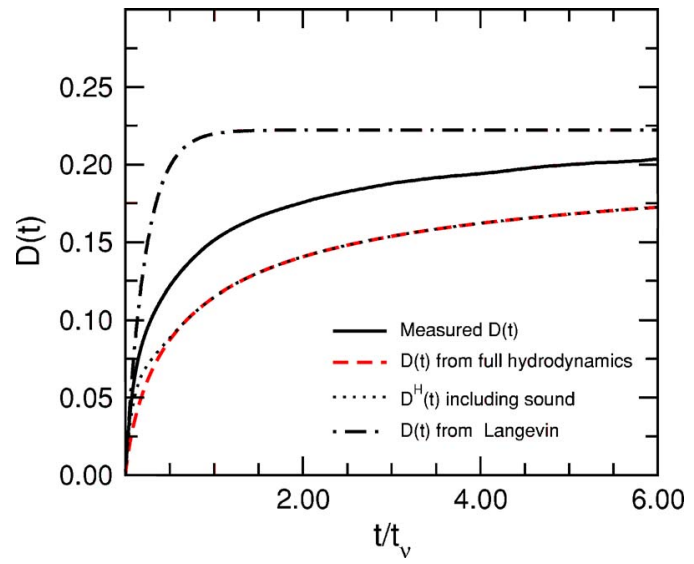

FIG. 14. (Color online) Integral $D(t)$ of the normalized VACF from Fig. 13 (solid line), compared to the integral of Eq. (B5) (dashed line) and including the contribution from sound as in Eq. (B14) (dotted line). The simple Langevin form from the integral of Eq. (B3) (dash-dotted line) is calculated with $\xi=6 \pi \eta \sigma_{c f}$ in order to have the same asymptotic value as $D^{H}(t)$. Because in the plot we integrate the normalized VACF, the $t \rightarrow \infty$ limit of $D(t)$ for the two theoretical approaches is $\frac{2}{9} t_{0}$ in our units, independent of colloid size. The measured $D(t)$ has a larger asymptotic value due to the initial effect of the Enskog friction.

$$
\lim _{t \rightarrow \infty}\langle V(t) V(0)\rangle^{H} \approx \frac{k_{B} T}{M_{c}} \frac{1}{9 \sqrt{\pi}}\left(\frac{\tau_{\nu}}{t}\right)^{3 / 2}=\frac{k_{B} T}{12 m \gamma(\pi \nu t)^{3 / 2}},
$$

with the same prefactor that was found from mode-coupling theory [99].

The integral of Eq. (B5) is

$$
\int_{0}^{\infty} d t\langle V(t) V(0)\rangle^{H}=\frac{k_{B} T}{6 \pi \eta a}
$$

which means that all the hydrodynamic contributions to the friction $\xi$ come from $\langle V(t) V(0)\rangle^{H}$. The integral converges slowly, as $\left(t / \tau_{\nu}\right)^{1 / 2}$, because of the long-time tail. The VACF can easily be related to the mean-square displacement, and deviations from purely diffusive motion are still observable for $t \gg \tau_{\nu}$ and have been measured in experiments $[98,100,101]$.

Although the last equality in Eq. (B6) shows that the VACF tail amplitude is independent of colloid size, it should be kept in mind that the algebraic decay does not set in until $t \approx \tau_{\nu}=a^{2} / \nu$. Thus it is the relative (not absolute) effect of the tail that is the same for all colloids. For example, at $t=\tau_{\nu}$, the VACF will have decayed to $4 \%$ of its initial value of $k_{B} T / M_{c}$, independently of colloid mass $M_{c}$. Even though this tail amplitude may seem small, about half the weight of the integral (B7) that determines the friction is from $t \geq \tau_{\nu}$. The dominance of the non-Markovian hydrodynamic behavior in determining the friction for colloids does not appear to hold for microscopic fluids, where-for example, for LennardJones liquids near the triple point-it is thought that the long-time tail only adds a few percent to the friction coeffi- 
TABLE IV. Summary of the physical properties of a 3D SRD fluid in the regime of small reduced mean free path $\lambda$. In the first column we show how these parameters scale for SRD. To highlight the scaling with the reduced mean free path $\lambda$ and the number of particles per cell, $\gamma$, we only show the collisional contribution to the viscosity in Eqs. (9)-(15), which dominates for small $\lambda$, and also ignore the factor $f_{\text {col }}^{\nu}(\gamma, \alpha)$. In the second column we list the parameter values for our choice of simulation parameters in this paper and in Refs. [52,58], and in the last column we compare to the parameter values for $\mathrm{H}_{2} \mathrm{O}$ at standard temperature and pressure. For the SRD the units are in terms of $m_{f}, a_{0}$, and $k_{B} T$, so that the time unit is $t_{0}=a_{0} \sqrt{m_{f} / k_{B} T}$.

\begin{tabular}{|c|c|c|c|c|}
\hline & & Scaling in SRD & Value for $\lambda=0.1, \gamma=5, \alpha=\frac{1}{2} \pi$ & Value for $\mathrm{H}_{2} \mathrm{O}$ \\
\hline$\rho_{f}$ & mass density & $\gamma \frac{m_{f}}{a_{0}^{3}}$ & $5 \frac{m_{f}}{a_{0}^{3}}$ & $1 \times 10^{-12} \mathrm{~g} / \mu \mathrm{m}^{3}$ \\
\hline$n_{f}$ & number density & $\gamma a_{0}^{-3}$ & $5 a_{0}^{-3}$ & $3.35 \times 10^{10} \mu \mathrm{m}^{-3}$ \\
\hline$c_{s}$ & speed of sound & $\sqrt{\frac{5 k_{B} T}{3 m_{f}}}$ & $1.29 \frac{a_{0}}{t_{0}}$ & $1.48 \times 10^{9} \mu \mathrm{m} / \mathrm{s}$ \\
\hline$\lambda_{\text {free }}$ & mean free path & $\lambda_{\text {free }}=\lambda a_{0}$ & $0.1 a_{0}$ & $\approx 3 \AA$ \\
\hline$\eta$ & shear viscosity & $\approx \frac{\lambda}{18 \lambda} \frac{m_{f}}{a_{0} t_{0}}$ & $\approx 2.50 \frac{m_{f}}{a_{0} t_{0}}$ & $1 \times 10^{-6} \mathrm{~g} / \mu \mathrm{m} \mathrm{s}$ \\
\hline$\nu$ & kinematic viscosity & $\approx \frac{1}{18 \lambda} \frac{a_{0}^{2}}{t_{0}}$ & $\approx 0.50 \frac{a_{0}^{2}}{t_{0}}$ & $1 \times 10^{6} \mu \mathrm{m}^{2} / \mathrm{s}$ \\
\hline$D_{f}$ & self-diffusion constant & $\approx \lambda \frac{a_{0}^{2}}{t_{0}}$ & $\approx 0.1 \frac{a_{0}^{2}}{t_{0}}$ & $2.35 \times 10^{3} \mu \mathrm{m}^{2} / \mathrm{s}$ \\
\hline$N_{\mathrm{Sc}}$ & Schmidt number & $\frac{\nu}{D_{f}} \approx \frac{1}{18 \lambda^{2}}$ & $\frac{\nu}{D_{f}} \approx 5$ & $\frac{\nu}{D_{f}} \approx 425$ \\
\hline
\end{tabular}

cient [102]. However, this is not due to the simple Langevin equation (B1) being more accurate, but rather to the modified short-time behavior of the VACF, caused by fluid correlations (a different non-Markovian effect).

\section{VACF at short times}

At short times the hydrodynamic contribution to the VACF (B5) reduces to

$$
\lim _{t \rightarrow 0}\langle V(t) V(0)\rangle^{H}=\frac{2}{3} \frac{k_{B} T}{M_{c}}
$$

because, within a continuum description, $\frac{1}{3}$ of the energy is dissipated as a sound wave at velocity $c_{s}$,

$$
\begin{aligned}
\langle V(t) V(0)\rangle^{c s}= & \frac{1}{3} \frac{k_{B} T}{M_{c}} \exp \left[-(3 / 2)\left(t / t_{c s}\right)\right] \\
& \times\left[\cos \left(\frac{\sqrt{3}}{2} \frac{t}{t_{c s}}\right)-\sqrt{3} \sin \left(\frac{\sqrt{3}}{2} \frac{t}{t_{c s}}\right)\right],
\end{aligned}
$$

where the sonic time $t_{c s}=a / c_{s}$ is defined in Table III. The sound wave does not contribute to the friction or diffusion since

$$
\int_{0}^{\infty} d t\langle V(t) V(0)\rangle^{c s}=0
$$

For a more extended discussion of the role of sound on hydrodynamics see, e.g., Ref. [89].

Because $t_{c s} \ll \tau_{\nu}$, the sound-wave contribution to the VACF decays much faster than the hydrodynamic contribution $\langle V(t) V(0)\rangle^{H}$ or even than the Langevin approximation $\langle V(t) V(0)\rangle^{L}$ (recall that $\tau_{\nu}=\frac{9}{2} \tau_{B}$ for neutrally buoyant colloids). Therefore, in the continuum picture, the short-time decay of the VACF is much faster than that suggested by the simple Langevin equation. And even after the sound wave has decayed, $\langle V(t) V(0)\rangle^{H}$ decays much more rapidly (faster than simple exponential) than Eq. (B3) for times $t \lesssim \tau_{B}$. SRD simulations confirm this more rapid short-time decay. For example, in Fig. 13 we show a direct comparison of the measured VACF to the continuum approach, where $\langle V(t) V(0)\rangle=\langle V(t) V(0)\rangle^{H}+\langle V(t) V(0)\rangle^{c s}$. For short times the decay predicted from sound agrees reasonable well, and for long times the full hydrodynamic theory quantitatively fits the simulation data.

A closer look at the short-time behavior in our SRD simulations, both for slip boundaries as in Fig. 10 and for stick 
TABLE V. Physical parameters, dimensionless hydrodynamic numbers, and time scales for spherical colloids of radius $a=0.01 \mu \mathrm{m}(10 \mathrm{~nm})$ and $a=1 \mu \mathrm{m}$ in $\mathrm{H}_{2} \mathrm{O}$ at standard temperature and pressure, moving at a velocity $v_{S}=10 \mu \mathrm{m} / \mathrm{s}$. The mass density $\rho_{c}$ of the colloid is taken to be the same as that of water (the colloid is neutrally buoyant), and the hydrodynamic radius $a$ is taken to be the same as the physical radius. Where, for notational clarity, the units are not explicitly shown, then length is measured in $\mu \mathrm{m}$, time in $\mathrm{s}$, and mass in $\mathrm{g}$.

\begin{tabular}{|c|c|c|}
\hline Physical parameters & $a=10 \mathrm{~nm}$ & $a=1 \mu \mathrm{m}$ \\
\hline$M_{c} \approx \frac{4}{3} \pi a^{3} \times 10^{-12} \mathrm{~g} / \mu \mathrm{m}^{3}$ & $4.19 \times 10^{-18} \mathrm{~g}$ & $4.19 \times 10^{-12} \mathrm{~g}$ \\
\hline$\frac{\xi_{E}}{\xi_{S}} \approx 1.6 \times 10^{2} a$ & 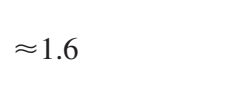 & $\approx 1.6 \times 10^{2}$ \\
\hline$\xi_{S}=6 \pi \eta a \approx 1.9 \times 10^{-5} a$ & $\approx 1.9 \times 10^{-7} \mathrm{~g} / \mathrm{s}$ & $\approx 1.9 \times 10^{-5} \mathrm{~g} / \mathrm{s}$ \\
\hline$D_{\mathrm{col}} \approx \frac{k_{B} T}{6 \pi \eta a} \approx \frac{0.2}{a}$ & $\approx 20 \mu \mathrm{m}^{2} / \mathrm{s}$ & $\approx 0.2 \mu \mathrm{m}^{2} / \mathrm{s}$ \\
\hline Hydrodynamic numbers & $a=10 \mathrm{~nm}$ & $a=1 \mu \mathrm{m}$ \\
\hline $\mathrm{P}_{\mathrm{e}}=\frac{v_{S} a}{D_{\mathrm{col}}} \approx 5 v_{S} a^{2}$ & $\approx 0.005$ & $\approx 50$ \\
\hline $\mathrm{R}_{\mathrm{e}}=\frac{v_{S} a}{\nu} \approx 10^{-6} v_{S} a$ & $\approx 10^{-7}$ & $\approx 10^{-5}$ \\
\hline $\mathrm{K}_{\mathrm{n}}=\frac{\lambda_{\text {free }}}{a} \approx \frac{0.3}{a} \times 10^{-3}$ & $\approx 0.03$ & $\approx 0.0003$ \\
\hline $\mathrm{M}_{\mathrm{a}}=\frac{v_{S}}{c_{s}} \approx 6.76 \times 10^{-10} v_{S}$ & $\approx 6.8 \times 10^{-10}$ & $\approx 6.8 \times 10^{-10}$ \\
\hline Time scales & $a=10 \mathrm{~nm}$ & $a=1 \mu \mathrm{m}$ \\
\hline$\tau_{D}=\frac{a^{2}}{D_{\mathrm{col}}} \approx 5 a^{3}$ & $\approx 5 \times 10^{-6} \mathrm{~s}$ & $\approx 5 \mathrm{~s}$ \\
\hline$t_{S}=\frac{a}{v_{S}}=\frac{\tau_{\nu}}{\mathrm{Re}}=\frac{\tau_{D}}{\mathrm{Pe}}$ & $=0.001 \mathrm{~s}$ & $=0.1 \mathrm{~s}$ \\
\hline$\tau_{\nu}=\frac{a^{2}}{\nu} \approx 10^{-6} a^{2}$ & $\approx 10^{-10} \mathrm{~s}$ & $\approx 10^{-6} \mathrm{~s}$ \\
\hline$\tau_{B}=\frac{M_{c}}{\xi_{S}}=\frac{2}{9} \tau_{\nu}$ & $\approx 2.2 \times 10^{-11} \mathrm{~s}$ & $\approx 2.2 \times 10^{-7} \mathrm{~s}$ \\
\hline$t_{c s}=\frac{a}{c_{s}} \approx 6.7 \times 10^{-10} a$ & $\approx 6.7 \times 10^{-12} \mathrm{~s}$ & $\approx 6.7 \times 10^{-10} \mathrm{~s}$ \\
\hline
\end{tabular}

boundaries as in Fig. 13 or in [58], shows that a better fit to the short-time exponential decay of the VACF is given by

$$
\langle V(t) V(0)\rangle^{E}=\frac{k_{B} T}{M_{c}} \exp \left(-t / \tau_{E}\right)
$$

where the Enskog time (53) scales as

$$
\tau_{E}=\frac{M_{c}}{\xi_{E}} \approx \frac{0.5 a}{\sqrt{k_{B} T / m_{f}}} \approx 0.645 t_{c s}
$$

for heavy (stick boundary) colloids in an SRD fluid. Note that the Enskog decay time is very close to the decay time $\frac{2}{3} t_{c s}$ of the sound wave (B9). That the SRD Enskog time should scale as $t_{c s}$ is perhaps not too surprising, because that is the natural time scale of the SRD fluid. However, the fact that the prefactors in the exponential of Eqs. (B9) and (B11) and are virtually the same may be accidental. Although in a real liquid the increased compressibility factor means $\tau_{E}$ and $\tau_{c s}$ are lower than their values for an ideal gas, the two times change at slightly different rates so that we do not expect the same equivalence in physical systems.

In contrast to the sound wave, the integral over the Enskog VACF leads to a finite value: 
TABLE VI. Physical parameters, dimensionless hydrodynamic numbers, and time scales for a stick boundary colloid in an SRD fluid. In the first column, we take the same small $\lambda$ limit as in Table IV to highlight the dominant scaling. In the other columns more accurate values for $\nu / \nu_{0}$ are used. The hydrodynamic numbers and times were estimated with a length scale $a \approx \sigma_{c f} \approx \sigma_{c c} / 2$. The colloids are neutrally buoyant, $\rho_{c}=\rho_{f}=\gamma m_{f} / a_{0}^{3}$, and their velocity is $v_{S}=0.05 a_{0} / t_{0}$-i.e., Ma $=0.039$. Units as in Table IV.

\begin{tabular}{|c|c|c|}
\hline Physical parameters & $\sigma_{c f}=2 a_{0}$ & $\sigma_{c f}=10 a_{0}$ \\
\hline$M_{c} \approx \frac{4}{3} \pi \gamma \sigma_{c f}^{3} \frac{m_{f}}{a_{0}^{3}}$ & $\approx 168 m_{f}$ & $2.09 \times 10^{4} m_{f}$ \\
\hline$\frac{\xi_{E}}{\xi_{S}} \approx 8.6 \sigma_{c f} \lambda$ & $\approx 1.8$ & $\approx 9.0$ \\
\hline$\xi_{S}=6 \pi \eta a \approx \frac{\lambda \sigma_{c f}}{\lambda}$ & $\approx 96 \frac{m_{f}}{t_{0}}$ & $\approx 478 \frac{m_{f}}{t_{0}}$ \\
\hline$D_{\mathrm{col}} \approx \frac{k_{B} T}{6 \pi \eta a} \approx \frac{\lambda}{\gamma \sigma_{c f}}$ & $\approx 0.0165 \frac{a_{0}^{2}}{t_{0}}$ & $\approx 0.00236 \frac{a_{0}^{2}}{t_{0}}$ \\
\hline Hydrodynamic numbers & $\sigma_{c f}=2 a_{0}$ & $\sigma_{c f}=10 a_{0}$ \\
\hline $\mathrm{P}_{\mathrm{e}}=\frac{v_{S} a}{D_{\text {col }}} \approx 1.29 \mathrm{Ma} \frac{\gamma \sigma_{c f}^{2}}{\lambda}$ & $\approx 6.2$ & $\approx 212$ \\
\hline $\mathrm{R}_{\mathrm{e}}=\frac{v_{S} a}{\nu} \approx 23 \mathrm{Ma} \lambda \sigma_{c f}$ & $\approx 0.2$ & $\approx 1$ \\
\hline $\mathrm{K}_{\mathrm{n}}=\frac{\lambda_{\text {free }}}{a} \approx \frac{\lambda}{\sigma_{c f}}$ & $\approx 0.05$ & $\approx 0.01$ \\
\hline $\mathrm{M}_{\mathrm{a}}=\frac{v_{S}}{c_{s}}$ & $\approx 0.039$ & $\approx 0.039$ \\
\hline Time scales & $\sigma_{c f}=2 a_{0}$ & $\sigma_{c f}=10 a_{0}$ \\
\hline$\tau_{D}=\frac{a^{2}}{D_{\mathrm{col}}} \approx \frac{\gamma \sigma_{c f}^{3}}{\lambda}$ & $\approx 242 t_{0}$ & $\approx 4.2 \times 10^{4} t_{0}$ \\
\hline$t_{S}=\frac{a}{v_{S}}=\frac{\tau_{\nu}}{\operatorname{Re}}=\frac{\tau_{D}}{\mathrm{Pe}} \approx \frac{\sigma_{c f}}{1.29 \mathrm{Ma}}$ & $\approx 31 t_{0}$ & $\approx 155 t_{0}$ \\
\hline$\tau_{\nu}=\frac{a^{2}}{\nu} \approx 18 \lambda \sigma_{c f}^{2}$ & $\approx 8.0 t_{0}$ & $\approx 200 t_{0}$ \\
\hline$\tau_{B}=\frac{\mathrm{M}_{\mathrm{c}}}{\xi_{S}} \approx \frac{2}{9} \tau_{v}$ & $\approx 1.75 t_{0}$ & $\approx 44 t_{0}$ \\
\hline$\tau_{E}=\frac{M_{c}}{\xi_{E}} \approx 0.5 \sigma_{c f}$ & $\approx 0.98 t_{0}$ & $\approx 4.9 t_{0}$ \\
\hline$t_{c s}=\frac{\sigma_{c f}}{c_{s}} \approx 0.78 \sigma_{c f}$ & $\approx 1.55 t_{0}$ & $\approx 7.8 t_{0}$ \\
\hline
\end{tabular}

$$
\int_{0}^{\infty} d t\langle V(t) V(0)\rangle^{E}=\frac{k_{B} T}{\xi_{E}}
$$

which quantitatively explains the friction we measured in our simulations, as discussed in Sec. V. In contrast to the VACF from sound, the Enskog result cannot simply be added to the
VACF from Eq. (B5) because this would violate the $t=0$ limit of the VACF, set by equipartition. However, because the Enskog contribution occurs on times $t \leqslant t_{c s}$, where sound and other collective modes of (compressible) hydrodynamics are not yet fully developed, it may be that Eqs. (B5) and (B9), derived from continuum theory, should be modified on these short time scales. Since the dominant contribution to 
the integral in Eq. (B7) is for longer times, the exact form of the hydrodynamic contribution (B5) on these short times $\left(t \lesssim t_{c s}\right)$ does not have much influence on the hydrodynamic part of the friction. Thus the approximation of the short-time VACF by the Enskog result of Eq. (B11) and the longer-time $\left(t \gg \tau_{E}\right.$, but still $t<\tau_{\nu}$ ) VACF by Eq. (B5) may be a fairly reasonable empirical approach. This approximation, together with the Green-Kubo relation (B4), then justifies, $a$ posteriori, the parallel addition of frictions in Eq. (41).

Just as was found for the long-time behavior, the shorttime behavior of the VACF displays important deviations from the simple Langevin picture. We find that the VACF decays much faster, on the order of the sonic time $\tau_{c S}$ rather than the Brownian time $\tau_{B}$. From Tables V and VI one can see how large these differences can be. For example, for colloids with $a=1 \mu \mathrm{m}$, the short-time Enskog or sonic decay times are of order $10^{-9} \mathrm{~s}$, while $\tau_{B}$ is of order $10^{-7} \mathrm{~s}$, so that the decay of the VACF will be dominated by $\langle V(t) V(0)\rangle^{H}$, with its nonexponential behavior, over almost the entire time regime.

An example of the effect of the Enskog contribution on the friction or the mean-square displacement is given in Fig. 14. The integral of the VACF, $D(t)$ from Eq. (58), which is directly related to the mean-square displacement [61], is extracted from the simulations described in Fig. 13 and compared to

$$
D^{H}(t)=\int_{0}^{t} d t^{\prime}\left[\left\langle V\left(t^{\prime}\right) V(0)\right\rangle^{H}+\left\langle V\left(t^{\prime}\right) V(0)\right\rangle^{c s}\right]
$$

calculated using Eqs. (B5) and (B9). Both show exactly the same $\left(t / \tau_{\nu}\right)^{1 / 2}$ scaling for $t \geqslant \tau_{\nu}$, but the simulated $D(t)$ is larger because the initial Enskog contribution means that $D(t) \approx D^{H}(t)+D_{E}$, for $t \gg \tau_{E}$, with $D_{E}=k_{B} T / \xi_{E}$ defined in Eq. (B13).

The slow algebraic convergence to the final diffusion coefficient, or related friction, also helps explain the scaling of the finite-size effects of Eq. (43). A finite box of length
$L$ means that the measured $D(t)$ is cut off roughly at $t_{L}=\left(L^{2} / a^{2}\right) \tau_{\nu}$, and because $\int d t D(t) \sim\left(\tau_{\nu} / t\right)^{1 / 2}$ for $t \gtrsim \tau_{\nu}$, the effect on the diffusion constant can be written as $D \approx D_{0}[1-c(L / a)]$ which explains the scaling form of Eq. (43). Figure 14 also demonstrates how much more rapidly the $D(t)$ from the Langevin equation (B3) converges to its final result $D=k_{B} T / \xi$ when compared to the simulations and hydrodynamic theories.

In summary, even though the simple Langevin equation (B1), without memory, may have pedagogical merit, it does a poor job of describing the VACF for colloids in solution.

\section{APPENDIX C: COMPARISON OF SRD TO PHYSICAL PARAMETERS}

At the end of the day one would like to compare a coarsegrained simulation to a real physical colloidal dispersion. To help facilitate such comparisons, we have summarized a number of physical parameters and dimensionless numbers for colloids in water, and also colloids in an SRD fluid.

Table IV compares the properties of a pure SRD fluid, in the limit of small mean free path $\lambda$, to the properties of water.

Table V summarizes the main scaling and values for a number of different properties of two different size neutrally buoyant colloids in water. To approximate the Enskog friction we used a simplified form valid for stick boundary conditions in an ideal fluid, as in [58], which ignores the compressibility and other expected complexities of real water. These predictions should therefore be taken with a grain of salt. For simplicity, we have ignored any potential Enskog effects on the diffusion constant or time-scales.

Table VI shows a very similar comparison for two different size colloids in an SRD fluid. For simplicity we assume that there are no finite-size effects, which explains small differences when compared to similar parameters used in the text of the main article. However, in contrast to Table V, we do include the Enskog contribution when calculating the total friction and related properties.
[1] J. K. G. Dhont, An Introduction to the Dynamics of Colloids (Elsevier, Amsterdam, 1996).

[2] W. B. Russel, D. A. Saville, and W. R. Showalter, Colloidal Dispersions (Cambridge University Press, Cambridge, England, 1989).

[3] D. L. Ermak, J. Chem. Phys. 62, 4189 (1975).

[4] D. L. Ermak and J. A. McCammon, J. Chem. Phys. 69, 1352 (1978).

[5] J. F. Brady and G. Bossis, Annu. Rev. Fluid Mech. 20, 111 (1988).

[6] A. Sierou and J. F. Brady, J. Fluid Mech. 448, 115 (2001).

[7] See, e.g., http://www.efluids.com/efluids/books/joseph.htm or http://www.aem.umn.edu/Solid-Liquid_Flows/for an overview.

[8] H. Tanaka and T. Araki, Phys. Rev. Lett. 85, 1338 (2000); Chem. Eng. Sci. 61, 2108 (2006).

[9] Y. Nakayama and R. Yamamoto, Phys. Rev. E 71, 036707
(2005).

[10] H. Tanaka (private communication).

[11] A. Succi, The Lattice Bolzmann Equation for Fluid Dynamics and Beyond (Oxford University Press, Oxford, 2001).

[12] A. J. C. Ladd, Phys. Rev. Lett. 70, 1339 (1993).

[13] A. J. C. Ladd and R. Verberg, J. Stat. Phys. 104, 1191 (2001).

[14] M. E. Cates et al., J. Phys.: Condens. Matter 16, S3903 (2004); R. Adhikaril, K. Stratford, M. E. Cates, and A. J. Wagner, Europhys. Lett. 71, 473 (2005); K. Stratford et al., Science 309, 2198 (2005).

[15] V. Lobaskin and B. Dünweg, New J. Phys. 6, 54 (2004); V. Lobaskin, B. Dünweg, and C. Holm, J. Phys.: Condens. Matter 16, S4063 (2004).

[16] F. Capuani, I. Pagonabarraga, and D. Frenkel, J. Chem. Phys. 121, 973 (2004).

[17] A. Chatterji and J. Horbach, J. Chem. Phys. 122, 184903 
(2005)

[18] P. J. Hoogerbrugge and J. M. V. A. Koelman, Europhys. Lett. 19, 155 (1992).

[19] P. Español and P. B. Warren, Europhys. Lett. 30, 191 (1995).

[20] A. A. Louis, P. G. Bolhuis, J. P. Hansen, and E. J. Meijer, Phys. Rev. Lett. 85, 2522 (2000).

[21] A. A. Louis, Philos. Trans. R. Soc. London, Ser. A 359, 939 (2001).

[22] C. N. Likos, Phys. Rep. 348, 267 (2001).

[23] S. H. L. Klapp, D. J. Diestler, and M. Schoen, J. Phys.: Condens. Matter 16, 7331 (2004).

[24] A. A. Louis, J. Phys.: Condens. Matter 14, 1987 (2002).

[25] J. T. Padding and W. J. Briels, J. Chem. Phys. 115, 2846 (2001); 117, 925 (2002).

[26] G. A. Vliegenthart and P. van der Schoot, Europhys. Lett. 62, 600 (2003).

[27] J. Dzubiella, H. Löwen, and C. N. Likos, Phys. Rev. Lett. 91, 248301 (2003).

[28] P. B. Warren, J. Chem. Phys. 15, S3467 (2003); see also I. Pagonabarraga and D. Frenkel, ibid. 115, 5015 (2001).

[29] B. Dünweg, in Computational Soft Matter: From Synthetic Polymers to Proteins, edited by N. Attig, K. Binder, H. Grubmuller, and K. Kremer, NIC Series, Volume 23, pp. 61 (Julich, 1994)

[30] T. Soddemann, B. Dunweg, and K. Kremer, Phys. Rev. E 68, 046702 (2003).

[31] P. B. Warren (private communication).

[32] V. Pryamitsyn and V. Ganesan, J. Chem. Phys. 122, 104906 (2005).

[33] C. P. Lowe, Europhys. Lett. 47, 145 (1999).

[34] C. P. Lowe, A. F. Bakker, and M. W. Dreischor, Europhys. Lett. 67, 397 (2004).

[35] A. Malevanets and R. Kapral, J. Chem. Phys. 110, 8605 (1999).

[36] G. A. Bird, Phys. Fluids 13, 2676 (1970).

[37] G. A. Bird, Molecular Gas Dynamics and the Direct Simulation of Gas Flows (Claredon Press, Oxford, 1994).

[38] T. Ihle and D. M. Kroll, Phys. Rev. E 63, 020201(R) (2001).

[39] T. Ihle and D. M. Kroll, Phys. Rev. E 67, 066705 (2003); 67, 066706 (2003).

[40] N. Kikuchi, C. M. Pooley, J. F. Ryder, and J. M. Yeomans, J. Chem. Phys. 119, 6388 (2003).

[41] C. M. Pooley and J. M. Yeomans, J. Phys. Chem. B 109, 6505 (2005).

[42] A. Lamura, G. Gompper, T. Ihle, and D. M. Kroll, Europhys. Lett. 56, 319 (2001).

[43] E. Allahyarov and G. Gompper, Phys. Rev. E 66, 036702 (2002).

[44] A. Malevanets and J. Yeomans, Europhys. Lett. 52, 231 (1999).

[45] M. Ripoll, K. Mussawisade, R. G. Winkler, and G. Gompper, Europhys. Lett. 68, 106 (2004).

[46] M. A. Webster and J. M. Yeomans, J. Chem. Phys. 122, 164903 (2005).

[47] N. Kikuchi, J. F. Ryder, C. M. Pooley, and J. M. Yeomans, Phys. Rev. E 71, 061804 (2005).

[48] H. Noguchi and G. Gompper, Phys. Rev. E 72, 011901 (2005).

[49] A. Malevanets and R. Kapral, J. Chem. Phys. 112, 7260 (2000).

[50] Y. Inoue, Y. Chen, and H. Obashi, J. Stat. Phys. 107, 85
(2002).

[51] S. H. Lee and R. Kapral, Physica A 298, 56 (2001).

[52] J. T. Padding and A. A. Louis, Phys. Rev. Lett. 93, 220601 (2004).

[53] M. Hecht, J. Harting, T. Ihle, and H. J. Herrmann, Phys. Rev. E 72, 011408 (2005).

[54] J. F. Ryder, Ph.D. thesis, Oxford University, 2005.

[55] T. Ihle, E. Tuzel, and D. M. Kroll, Phys. Rev. E 72, 046707 (2005).

[56] M. Ripoll, K. Mussawisade, R. G. Winkler, and G. Gompper, Phys. Rev. E 72, 016701 (2005).

[57] E. Guyon, J.-P. Hulin, L. Petit, and C. D. Mitescu, Physical Hydrodynamics (Oxford Univeristy Press, Oxford, 2001).

[58] J. T. Padding, A. Wysocki, H. Löwen, and A. A. Louis, J. Phys.: Condens. Matter 17, S3393 (2005).

[59] J. C. Maxwell, in The Scientific Papers of James Clerk Maxwell, edited by W. D. Niven (Dover, New York, 1965), Vol. 2.

[60] A. Wysocki, H. Löwen, J. T. Padding, and A. A. Louis (unpublished).

[61] J. P. Hansen and I. R. McDonald, Theory of Simple Liquids, 2nd ed. (Academic Press, London, 1986).

[62] M. P. Allen and D. J. Tildesley, Computer Simulation of Liquids (Clarendon Press, Oxford, 1987).

[63] J. Happel and H. Brenner, Low Reynolds Number Hydrodynamics: With Special Applications to Particulate Media (Springer, New York, 1983).

[64] L. Bocquet and J.-L. Barrat, Phys. Rev. E 49, 3079 (1994).

[65] J.-L. Barrat and L. Bocquet, Phys. Rev. Lett. 82, 4671 (1999).

[66] J. L. Lebowitz and H. Spohn, J. Stat. Phys. 19, 633 (1978).

[67] S. Asakura and F. Oosawa, J. Polym. Sci., Polym. Symp. 33, 183 (1958); A. Vrij, Pure Appl. Chem. 48, 471 (1976).

[68] A. A. Louis, E. Allahyarov, H. Löwen, and R. Roth, Phys. Rev. E 65, 061407 (2002).

[69] J. Vermant and M. J. Solomon, J. Phys.: Condens. Matter 17, R187 (2005).

[70] A. A. Potanin and W. B. Russel, Phys. Rev. E 52, 730 (1995).

[71] P. A. Nommensen, M. H. G. Duits, D. van den Ende, and J. Mellema, Phys. Rev. E 59, 3147 (1999).

[72] J. R. Melrose and R. C. Ball, J. Rheol. 48, 937 (2004).

[73] N.-Q. Nguyen and A. J. C. Ladd, Phys. Rev. E 66, 046708 (2002).

[74] Note that one could also define the volume fraction using an effective hard-sphere radius derived from the BarkerHenderson or other related criteria [61].

[75] J.-P. Matas, J. F. Morris, and E. Guazzelli, Phys. Rev. Lett. 90, 014501 (2003).

[76] D. R. Mikulencak and J. F. Morris, J. Fluid Mech. 520, 215 (2004).

[77] E. Purcell, Am. J. Phys. 45, 3 (1977).

[78] H. C. Berg, Random Walks in Biology (Princeton University Press, Princeton, 1993).

[79] A. J. C. Ladd, Phys. Rev. Lett. 76, 1392 (1996); 88, 048301 (2002).

[80] Y. Zhang, R. Qin, and D. R. Emerson, Phys. Rev. E 71, 047702 (2005), and references therein.

[81] T. S. Squires and S. R. Quake, Rev. Mod. Phys. 77, 977 (2005).

[82] G. K. Batchelor, J. Fluid Mech. 56, 375 (1972).

[83] J. T. Hynes, Annu. Rev. Phys. Chem. 28, 301 (1977); J. T. Hynes, R. Kapral, and M. Weinberg, J. Chem. Phys. 70, 1456 
(1979)

[84] S. H. Lee and R. Kapral, J. Chem. Phys. 121, 11163 (2004).

[85] A. A. Zick and G. M. Homsy, J. Fluid Mech. 115, 13 (1982).

[86] B. Dünweg and K. Kremer, J. Chem. Phys. 99, 6983 (1993).

[87] J. R. Schmidt and J. L. Skinner, J. Chem. Phys. 119, 8062 (2003).

[88] S. Henderson, S. Mitchell, and P. Bartlett, Phys. Rev. Lett. 88, 088302 (2002).

[89] A. F. Bakker and C. P. Lowe, J. Chem. Phys. 116, 5867 (2002).

[90] B. Cichocki and B. U. Felderhof, J. Chem. Phys. 89, 3705 (1988).

[91] M. Hecht (private communication).

[92] G. A. Vliegenthart and H. N. W. Lekkerkerker, J. Chem. Phys. 112, 5364 (2000).

[93] A. J. Hurd, N. A. Clark, R. C. Mockler, and W. J. O’Sullivan, Phys. Rev. A 26, 2869 (1982).
[94] B. J. Alder and T. E. Wainwright, Phys. Rev. A 1, 18 (1970).

[95] E. H. Hauge and A. Martin-Löf, J. Stat. Phys. 7, 259 (1973).

[96] E. J. Hinch, J. Fluid Mech. 72, 499 (1975).

[97] V. Lisy and J. Tothova, e-print cond-mat/0410222, point out that very similar results to $[95,96]$ were obtained in the Russian scientific literature, most notably V. Vladimirsky and Ya. Terletzky, Zh. Eksp. Teor. Fiz. 15, 259 (1945); A. V. Zatovsky, Izv. Vuzov. Fizika 10, 13 (1969).

[98] G. L Paul and P. N. Pusey, J. Phys. A 14, 3301 (1981).

[99] M. H. Ernst, E. H. Hauge, and J. M. J. van Leeuwen, Phys. Rev. Lett. 25, 1254 (1970).

[100] D. A. Weitz, D. J. Pine, P. N. Pusey, and R. J. A. Tough, Phys. Rev. Lett. 63, 1747 (1989).

[101] B. Lukić, S. Jeney, C. Tischen, A. J. Kulik, L. Forro, and E. L. Florin, Phys. Rev. Lett. 95, 160601 (2005).

[102] D. Levesque and W. T. Ashurst, Phys. Rev. Lett. 33, 277 (1974). 